【光电工程／Optoelectronic Engineering】

\title{
操纵微纳颗粒的“光之手”一一光镊技术研究进展
}

闵长俊，袁运琪，张聿全，汪先友，张治斌，袁小聪

深圳大学纳米光子学研究中心, 深圳市微尺度光信息重点实验室, 微纳光电子学研究院, 广东深圳 518060

摘 要: 光铝是操纵微纳米尺寸颗粒的重要技术手段，已在物理、化学、生物及医学等领域得到广泛 应用. 综述近年来光铌领域的研究进展, 系统介绍光铒研究的主要方向及代表性成果. 介绍了光铝技术的 理论基础，包括光铝捕获物体的作用机理和几种常用理论模型; 按照产生光铝的光束类型将光铝技术分为 结构光束光铝、多光束与全息光铌、近场修逝波光铝、表面等离激元光铌、光纤光镊、热电光铝、飞秒激 光光镊及异常光学力光铌, 分别介绍各类光镊技术的特点和最新进展; 介绍光铒技术在生物样品操纵、分 子检测及裸眼三维显示等领域的创新应用，并对光铝技术发展进行总结和展望.

关键词: 光学; 光铌; 光学全息; 矢量光束; 表面等离激元; 光纤光铒; 表面增强拉曼散射; 三维显示

中图分类号：0436 文献标志码：A doi：10.3724/SP. J.1249.2020.05441

\section{The hand of light for micro/nano-particle manipulation: research progress of optical tweezers}

\author{
MIN Changjun, YUAN Yunqi, ZHANG Yuquan, WANG Xianyou, \\ ZHANG Zhibin, and YUAN Xiaocong
}

\author{
Nanophotonics Research Center, Shenzhen Key Laboratory of Micro-Scale Optical Information Technology, \\ Institute of Microscale Optoelectronics, Shenzhen University, Shenzhen 518060, Guangdong Province, P. R. China
}

\begin{abstract}
Optical tweezer is an important technology for trapping and manipulating micro/nano-particles, which has widely been applied in physics, chemistry, biology, medicine and other fields. In this work, based on the research progresses in recent years, we systematically review the main research directions and representative achievements of optical tweezers. We introduce the theoretical basis about the mechanism of capturing objects and several common theoretical models of optical tweezers. According to the distribution of incident light beams, we classify the optical tweezers into structural beam optical tweezer, multi-beam and holographic optical tweezer, near-field evanescent wave tweezer, surface plasmon optical tweezer, optical fiber tweezer, opto-thermoelectric tweezer, femtosecond laser tweezer, and extraordinary optical forces tweezer. And the characteristics and latest developments of optical tweezers are discussed in detail respectively. In terms of innovative applications, we emphatically focus in the fields of biological sample manipulation, molecular detection, naked-eye three dimensional display, etc. In the final section, we provide a summary and prospect about the future development of optical tweezers technology.
\end{abstract}

Key words: optics; optical tweezers; optical holography; vector beam; surface plasmon; fiber optical tweezers; surface-enhanced Raman scattering; three dimensional display

Received: 2020-02-27; Accepted: 2020-05-18

Foundation: National Natural Science Foundation of China $(91750205,61935013,61975128)$

Corresponding author: Professor YUAN Xiaocong. E-mail: xcyuan@ szu.edu.cn

Citation: MIN Changjun, YUAN Yunqi, ZHANG Yuquan, et al. The hand of light for micro/nano-particle manipulation: research progress of optical tweezers [J]. Journal of Shenzhen University Science and Engineering, 2020, 37 (5): 441-458. (in Chinese) 
光是探索世界的重要手段，人类对光的研究一 直在不断深人, 从经典光学到现代光学, 研究方向 从光的自身特性逐渐扩展到光与物质的相互作用. 麦克斯韦在 19 世纪就基于光的电磁波理论证明光 不仅具有能量, 而且具有动量, 可对物质产生力学 作用. 然而, 普通光源的能量密度低, 不足以让光 的动量产生显著效应. 直到 1960 年激光器的发明 才使光的动量有了用武之地, 推动相关实验研究的 进展. 1986 年 ASHKIN 等 ${ }^{[1]}$ 利用单束激光聚焦实现 溶液中介质微球的三维捕获, 开创了光镊研究的先 河. 光镊是基于光与物质之间动量传递产生的一种 对微纳米尺寸颗粒捕获和操纵的方法, 具有非接触 和无损伤等优点, 对生物、物理及材料等领域研究 具有重要价值 ${ }^{[2-4]}$. 2018 年 ASHKIN 因发明光镊技 术获得诺贝尔物理学奖，在国际上激发了越来越多 的研究兴趣, 并推动新型光铌技术及其创新应用的 出现. 本文综述光镊技术的最新研究进展, 介绍光 镊相关经典理论模型、光镊类型及其特点、代表性 的光铌技术应用，最后对光铌技术及其应用的发展 趋势进行前瞻.

\section{1 光镊技术的理论基础}

光镊是利用一束光实现对微小物体的捕获. 为 了对物体产生捕获效果，必须使光在空间中产生一 个势能最低点, 形成力学势阱. 当该势阱的势垒大 于物体动能时，物体会被稳定束缚在势吽中.

光铌可以使物体在二维平面和三维空间中被捕 获. 典型的光铌三维捕获过程如图 1(a)，一束激 光经过透镜聚焦之后，与上方焦点附近的透明介质 小球发生相互作用，包括光在小球表面的反射、小 球内部的折射和吸收等，使得出射光的总动量发生 变化. 由动量守恒定律和牛顿第二定律可知, 光的 总动量随时间的变化率数值上等于小球所受到的光 学力, 主要包括光束对小球的推力和拉力. 其中, 推力主要来自于小球对光的反射和吸收, 这使得光 的动量传递给小球，从而把小球沿光的传播方向推 动, 通常也被称为辐射压力或散射力, 如图 1 (a) 中 $F_{\text {scat }}$. 拉力部分主要来自于小球对光的折射以及 高强度光焦点对小球的电荷极化作用，会形成势阱 把小球拉向光场最强点 (焦点) 并束缚起来，其力的 大小和聚焦光场的光强梯度分布有关，也被称为梯 度力, 如图 1 (a) 中 $F_{\text {grad }}{ }^{[5]}$. 小球受光场中 $F_{\text {scat }}$ 和 $F_{\text {grad }}$ 的共同作用，当 $F_{\text {scat }}<F_{\text {grad }}$ 时，就可以被捕获
在三维光场的焦点附近. 光镊捕获通常发生在溶液 中, 小球的重力和浮力相互抵消后与光学力相比非 常微弱, 可以忽略不计.

近年来，光镊的二维捕获因为可以应用于片上 光学系统, 受到越来越多的关注. 图 1 (b) 为光铌 二维捕获过程示意图，其中，人射激光在下层介质 基底和上层水溶液分界面处发生全反射，在溶液中 产生光学修逝波 ${ }^{[6]}$. 修逝波范围内的颗粒，在垂直 方向由于修逝波光强迅速衰减而受到指向基底的光 学梯度力, 在水平方向受到沿光束传播方向的散射 力. 通过合适的聚焦光束设计，可将水平方向散射 力抵消，从而使颗粒主要受垂直方向梯度力的作用 被捕获在界面附近. 由于修逝波离开界面后强度迅 速衰减，在溶液中垂直方向穿透深度一般小于人射 光波长，因此，该光镊技术只能在基底表面的二维 平面范围内形成颗粒捕获，适用于颗粒的片上操纵 与检测.

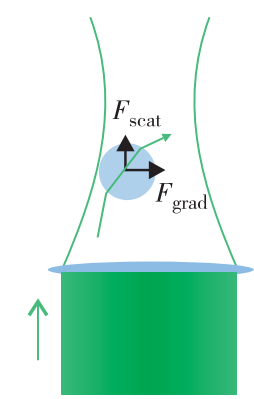

(a ) 光铌三维捕获示意图

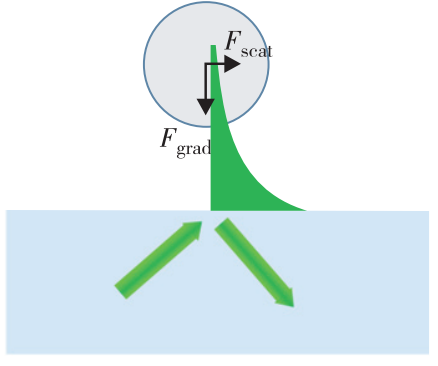

（b）光铌二维捕获的示意图

\section{图 1 光镊捕获示意图}

Fig. 1 Optical tweezers trapping schematic

在光镊的力学计算与分析中，可以根据尺寸大 小，将捕获的颗粒分成 3 类处理. 第 1 类为尺寸远 大于人射光波长的米氏 (Mie) 颗粒，可以直接利用 几何光学模型, 基于出射光的传播方向变化和动量 守恒定律，对颗粒所受光学力进行分析 ${ }^{[7]}$. 第 2 类 为尺寸远小于光波长 (一般在波长的 $1 / 10$ 以下) 的 颗粒，称为瑞利 (Rayleigh) 颗粒，其对光的作用满 足瑞利散射理论, 可将颗粒作为电偶极子进行受力 分析 ${ }^{[8]}$. 其中, 散射力主要由电偶极子对光的吸收 和再辐射引起, 力的大小与人射光强、环境介质折 射率、颗粒半径和颗粒折射率有关，力的方向为光 束波矢方向. 散射力 $F_{\text {scat }}{ }^{[9]}$ 为

$$
F_{\text {scat }}=\frac{I_{0} n_{\mathrm{m}}}{\mathrm{c}} \frac{128 \pi^{5} a^{6}}{3 \lambda^{4}}\left(\frac{m^{2}-1}{m^{2}+2}\right)^{2}
$$

其中, $I_{0}$ 为人射到颗粒上的光强; $n_{\mathrm{m}}$ 为颗粒周围介 质的折射率; $\mathrm{c}$ 为真空中的光速; $a$ 为颗粒半径; $\lambda$ 
为人射光波长; $m$ 为颗粒与周围介质折射率的比值. 梯度力由电偶极子与光场之间的电磁场力经过时间 平均得到, 力的大小与颗粒位置的光强梯度成正 比. 梯度力 $F_{\text {grad }}{ }^{[9]}$ 为

$$
F_{\text {grad }}=\frac{n_{m}^{2} a^{3}}{2}\left(\frac{m^{2}-1}{m^{2}+2}\right)^{2} \nabla I_{0}
$$

当焦点形成的轴向梯度力与散射力平衡, 同时光束 截面内梯度力势阱大于热运动动能时，可以形成稳 定的三维捕获. 第 3 类为尺寸与人射光波长相近的 颗粒，称为介观颗粒. 此类颗粒通过洛伦兹-米氏 散射模型 ${ }^{[10-12]}$, 将颗粒与光的作用作为电磁散射问 题进行处理，再通过计算散射后电磁场对颗粒产生 的洛伦兹力进行受力分析. 具体过程首先基于麦克 斯韦方程组求解颗粒散射后的电磁场分布，可采用 的数值模拟方法包括时域有限差分法、有限元法、 离散偶极子法及 $\mathrm{T}$ 矩阵法等 ${ }^{[12-15]}$; 再把计算获得的 颗粒周围电磁场分布代人麦克斯韦应力张量矩阵, 计算颗粒所受电磁场力的大小和方向. 根据麦克斯 韦应力张量矩阵, 颗粒所受的总电磁场力 ${ }^{[16]}$ 为

$$
\begin{aligned}
\langle F\rangle= & \oint_{S}\left\{\frac{\varepsilon}{2} \operatorname{Re}\left[(\boldsymbol{E} \cdot \boldsymbol{n}) \boldsymbol{E}^{*}\right]-\frac{\varepsilon}{4}\left(\boldsymbol{E} \cdot \boldsymbol{E}^{*}\right) n+\right. \\
& \left.\frac{\mu}{2} \operatorname{Re}\left[\mu(\boldsymbol{H} \cdot \boldsymbol{n}) \boldsymbol{H}^{*}\right]-\frac{\mu}{4}\left(\boldsymbol{H} \cdot \boldsymbol{H}^{*}\right) \boldsymbol{n}\right\} \mathrm{d} S
\end{aligned}
$$

其中, $\langle F\rangle$ 表示时间平均后的电磁场力; $E$ 为电场矢 量; $\boldsymbol{H}$ 为磁场强度; $\varepsilon$ 为颗粒周围介质的介电常数; $\mu$ 为颗粒周围介质的磁导率; $S$ 面为任意包含散射颗 粒的封闭曲面; $\boldsymbol{n}$ 为垂直于颗粒表面小面元 $\mathrm{d} S$ 的法 向单位矢量. 该方法的适用性最广，可用于计算不 同形状和材料的颗粒在电磁场中的受力情况.

此外, 也采用光阱刚度标定光铌系统的捕获能 力. 光阱刚度定义为颗粒受到的光阱捕获力与颗粒 偏离光阱中心的距离之比. 光阱刚度越大, 说明在 相同距离颗粒所受的光学捕获力越强. 光吽刚度也 是利用光镊进行微小力测量时所需的重要参数.

\section{2 光镊技术的研究进展}

光镊技术在研究人员的不断探索下展现出旺盛 的生命力. 近年来随着光场调控技术与器件的迅速 发展, 新型聚焦光场的产生使得新型光镊技术不断 涌现, 出现新奇的颗粒捕获与操控效果. 以下按照 产生光镊的不同光束类型，将光镊技术分为结构光 束光镊、多光束与全息光镊、近场修逝波光镊、表 面等离激元光镊、光纤光镊、热电光镊、飞秒激光
光镊及异常光学力光镊, 分别综述其原理及代表性 成果.

\section{1 结构光束光铌}

不同种类的激光光束都能产生光镊 ${ }^{[17]}$, 早期 传统光镊技术一般采用单束激光聚焦产生, 光束强 度分布为高斯型, 相位分布为均匀相位, 偏振分布 在光束截面内是均匀的 (称为标量偏振光束). 产生 光镊的标量偏振光束有线偏振光、圆偏振光和混合 偏振光. 线偏振光由于其单一方向偏振的特点, 所 产生聚焦场具有一定指向性, 在光镊中可应用于纳 米线或多颗粒样品的方向控制 ${ }^{[18]}$. 圆偏振光的特 点是光传播过程中电矢量振动方向发生旋转, 所以 带有自旋角动量, 在光镊中不但可以捕获颗粒, 还 可以使空间结构不均匀的颗粒产生自转 ${ }^{[19-20]}$. 圆偏 振光在高数值孔径物镜的紧聚焦条件下产生自旋角 动量到轨道角动量的转化, 即自旋-轨道耦合效 应 $^{[21]}$, 从而在聚焦场产生光学旋浴, 使所捕获颗 粒绕着光轴产生公转 ${ }^{[22]}$. 混合偏振光包括部分偏 振光和椭圆偏振光, 可以近似看成线偏光和圆偏光 的叠加, 也都可以用来产生光镊, 实现对颗粒的捕 获.

近些年，随着光场调控技术的发展，对光偏振 和相位的调控手段更加多样化, 产生许多偏振和相 位在空间上非均匀分布的光束 (称为结构光束), 包括偏振非均匀分布的矢量光束, 如径向偏振光和 角向偏振光 ${ }^{[23]}$; 相位非均匀分布光束, 如光学旋 浴光束 ${ }^{[24-25]}$ 和艾里 (Airy) 光束 ${ }^{[24-25]}$; 偏振和相位同 时非均匀分布的光束, 如带有圆偏振、径向或角向 偏振的光学旋浴. 这些结构光束在聚焦条件下具有 新颖特性, 为光镊技术发展带来新活力.

径向偏振光在传播过程中, 光束截面内任意位 置的电矢量振动方向都沿径向方向, 因此, 光轴中 心为偏振奇点, 导致光轴的光强度为 0 , 光强呈环 形分布. 径向偏振光在紧聚焦条件下焦点尺寸比线 偏振光和圆偏振光更小, 且具有更强的轴向电场分 量, 其产生的光铌捕获瑞利型金属颗粒的能力更 强, 且具有更好的轴向捕获能力 ${ }^{[26-28]}$. 角向偏振光 与径向偏振光在偏振方向上完全正交, 其光束截面 内电矢量振动均沿方位角方向, 光轴中心为偏振奇 点, 光强为环形分布. 基于角向偏振光的光镊在紧 聚焦条件下具有更高的横向捕获效率, 如图 2 (a).

光学旋涡光束的波前呈旋涡状, 这是由于其包 含螺旋相位因子, 并携带轨道角动量, 其螺旋相位 的分布与轨道角动量的拓扑荷数相关. 基于光学旋 
浴的光镊所携带的轨道角动量能够传递给所捕获的 颗粒，从而对颗粒产生一个旋转力矩的作用，使颗 粒围绕光轴旋转, 旋转的速度和方向可通过轨道角 动量的拓扑荷数进行调控 (如图 $2(b)-(d))$, 光 束传播方向垂直纸面向外，因此，这类光镊也被称 为光学扳手 ${ }^{[29-30]}$. 艾里光束由傍轴衍射方程的一个 无衍射特解得来, 可通过 3 次相位器件产生, 具有 弯曲轨道传播、无衍射及自重构的特性 ${ }^{[31]}$. 基于 艾里光束的光镊具有以下特点: (1) 弯曲轨道传播 特性可以操纵颗粒沿弯曲轨道移动, 从而避开障碍 物; (2) 无衍射性使光束发散变小, 可在纵向保持 较长的颗粒操纵范围; (3) 自重构性使颗粒散射后 的光场得以恢复原状，可用于多平面和多颗粒的捕
获和操纵. 艾里光束作用下颗粒向主光瓣方向移动,

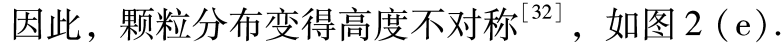

在偏振和相位均为非均匀分布的光束中，圆偏 振的光学旋浴光镊比线偏振、径向或角向偏振情况 可提供更大的颗粒旋转力矩 ${ }^{[33]}$ ，同时在这种光束 光镊中不仅可以使颗粒做轨道运动, 还可以使其做 自旋运动 ${ }^{[34]}$.

由于偏振和相位调控的多样性, 研究也提出更 多新颖的结构光束，将进一步增强光镊捕获颗粒的 能力，带来更加丰富的颗粒操控效果 ${ }^{[35]}$. 但目前 该技术受到结构光束产生器件和方法的制约, 存在 光束产生效率低、光束纯度不高及系统复杂等缺 点，有待进一步发展和突破.
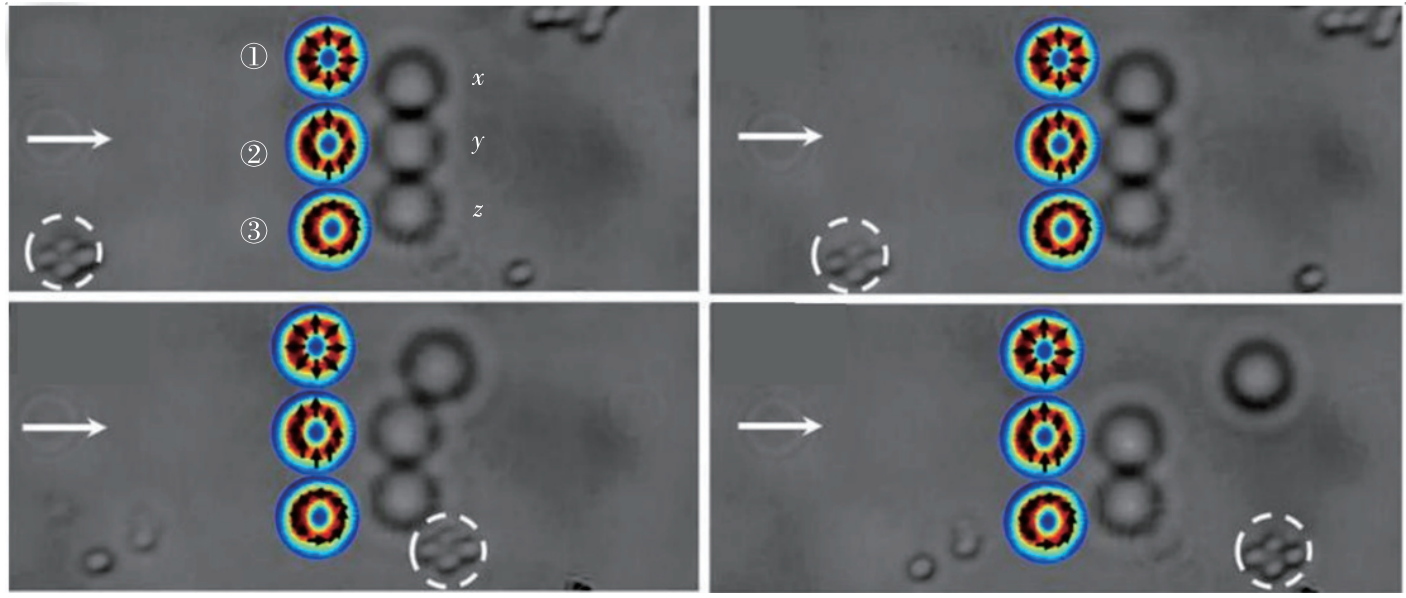

(a ) 由径向、线性和角向偏振光组成的多矢量光束光铌对直径 $2 \mu \mathrm{m}$ 二氧化硅微球进行移动 ${ }^{[36}$
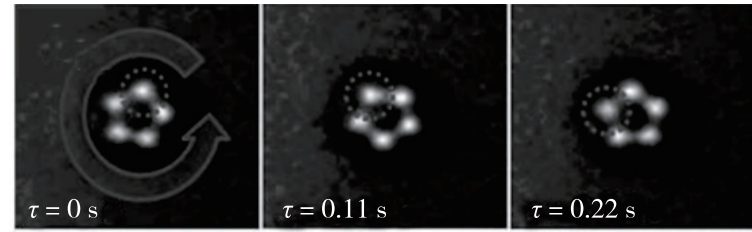

(b) 捕获光束为右旋光学旋涡时颗粒逆时针旋转

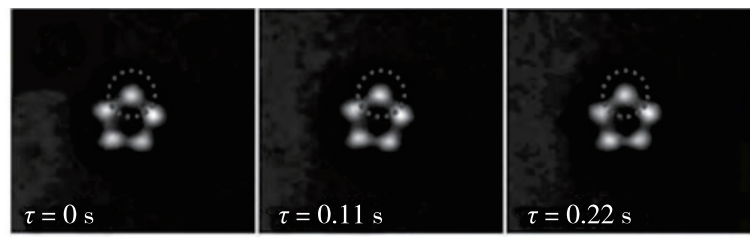

(c) 捕获光束无旋浴时颗粒不旋转 [37
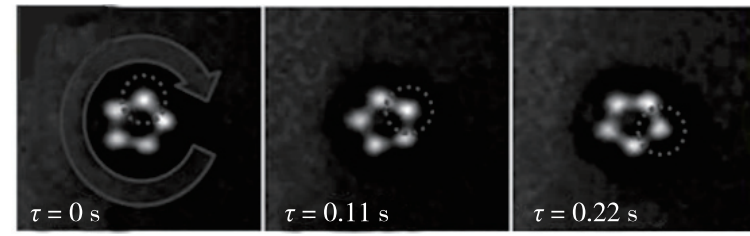

(d) 捕获光束为左旋光学旋浴时颗粒顺时针旋转 [37

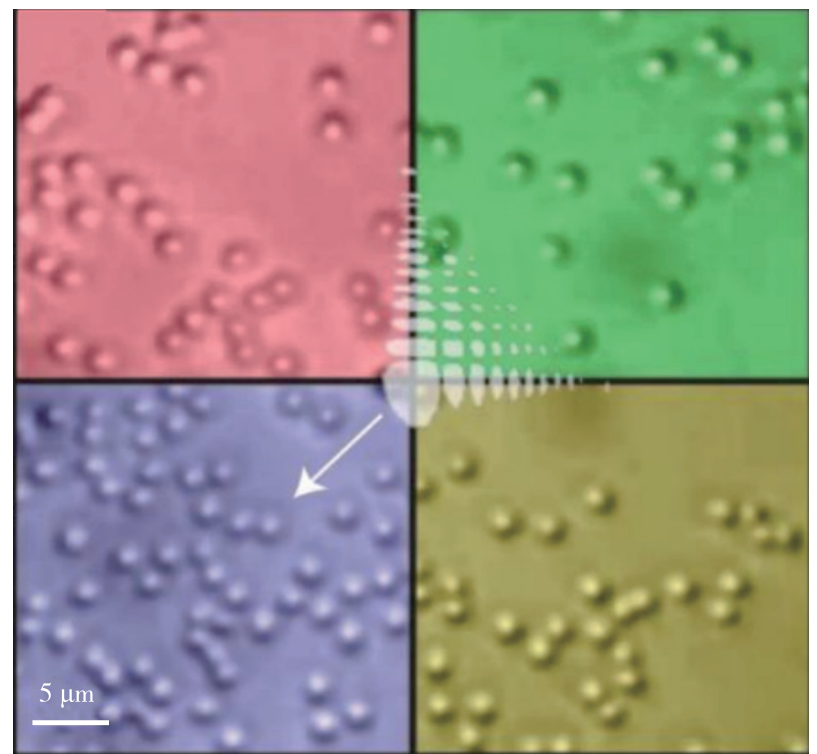

（e）艾里光束对介质颗粒施加光学梯度力， 将其拖向主瓣方向 ${ }^{[32]}$

图 2 结构光束光镊

Fig. 2 Structural beam optical tweezers 


\section{2 多光束与全息光铒}

光镊最初由单束激光聚焦产生, 单光束光镊的 简单性和稳定性对于研究光镊技术原理和开发新应 用方向具有重要意义，往往是各类光镊技术创新研 究的首选. 然而, 单光束可调控的自由度有限, 为 了增加调控自由度, 实现更复杂和多样化的功能, 逐渐发展出多光束光镊和全息光镊技术.

多光束光镊有效组合多个光束形成光镊系统, 比单光束光镊具有更高的操纵自由度和稳定性. 多 光束的产生方法包括：(1) 使用二向色镜进行多光 束分解和叠加, 通过偏振元件分离单个光束产生多 光束 ${ }^{[38]}$; (2) 单光束快速刷新扫描得到多个独立光 束 ${ }^{[39]}$, 通过液晶空间光调制器或数字微镜器件产 生多光束等. 多光束光镊系统既可以利用几束光共 同捕获单个颗粒，提升单颗粒的捕获精度和稳定 性，也可以分别捕获和操纵多个颗粒，便于研究颗 粒之间的相互作用，极大拓展了光镊的应用范围. 多光束光镊技术在原子物理学领域也有广泛应用, 代表之一就是利用多束激光共同作用捕获和冷却单

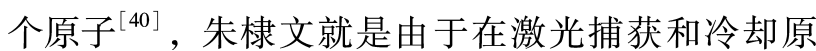
子方面的贡献获得 1997 年诺贝尔物理学奖.

全息光镊是利用全息光学元件产生图案化的全 息光场，进而实现更复杂的光铌捕获效果. 其实现 方式通常是把设计好的全息图加载到液晶空间光调 制器或数字微镜器件等动态调制器件, 产生具有多 个焦点的全息光场，实现多个颗粒的三维光镊捕获 与操纵 ${ }^{[41]}$. 基于这些全息光学元件, 可以通过设 计不同全息图，对光束的振幅和相位进行调制，因 此，在理论上可以产生任意形状、大小和数量的焦 点和光阱分布 ${ }^{[42-43]}$, 具有极高的自由度. 利用全息 光铌可以捕获多个颗粒排列成环形和阵列等任意图 案(图 3), 在多种不同颗粒分选及颗粒微结构加工 等领域具有重要应用价值.

多光束及全息光铌技术还可以与结构光束结 合，构建更复杂的全息结构光场，如 $\mathrm{YU}$ 等 ${ }^{[44]}$ 提出 使用液晶空间光调制器产生三维艾里光束阵列的全 息方法, 可实现颗粒阵列的产生与操控, 拓展了光 镊技术的应用范围. 该技术目前需要进一步提升全 息光学器件的效率，使人射光分散为复杂全息光场 后，仍可以形成足够强的颗粒捕获光阱.

\section{3 近场修逝波光铌}

在图 1 (b) 所示的光镊二维捕获中，通过全内 反射方法可以在界面近场范围内产生光学修逝波, 修逝波强度在远离界面的方向迅速衰减，因此，在

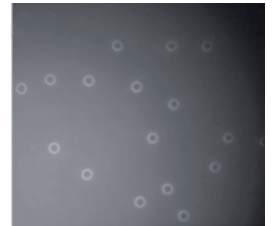

（a）使用7个全息阱 群将 24 个随机分布 的 $4.2 \mu \mathrm{m}$ 直径聚苯 乙烯微球分别捕获

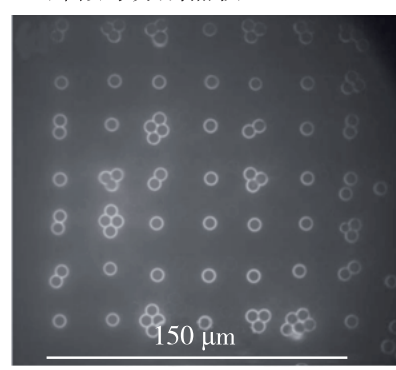

(d) $7 \times 7$ 宽场产生的光阱阵列, 用于捕获流经样品室的 $6 \mu \mathrm{m}$ 直径聚苯乙烯微球

图 3 全息光镊 ${ }^{[42]}$

Fig. 3 Holographic optical tweezers ${ }^{[42]}$

垂直于界面方向有很大的光强梯度变化，从而产生 很强的光学梯度力用于颗粒捕获，这就是近场修逝 波光铝技术的原理.

近场修逝波光镊的结构主要有全反射结构和波 导结构. 其中，全反射结构根据光源人射方式不同 又可以分为棱镜全内反射法和聚焦全反射法. 这两 种方法的原理近似，都是利用大角度人射光从光密 介质进人光疏介质时发生全内反射，在反射界面附 近产生光学修逝场. 两者区别在于棱镜全内反射法 一般用两束光从左右两侧分别斜人射三角形棱镜 （如图 4 (a)），在棱镜上表面处产生纵修逝场干涉 条纹 ${ }^{[45]}$, 从而抵消水平方向的散射力 (图 $\left.1(b)\right)$, 增强垂直方向的梯度力，最终用于颗粒的二维捕 获，如图 4 (b) ; 聚焦全反射法通过高数值孔径的 物镜聚焦人射光束，同时在物镜前遮挡人射光中心 部分来消除小于全内反射角的人射光，只保留大于 全内反射角的人射光在界面形成聚焦的修逝场，并 利用修逝场焦点捕获颗粒 ${ }^{[46-48]}$.

波导结构的近场修逝波光镊利用光在波导内传 播时，在波导界面反射产生修逝波的特性，将颗粒 捕获在波导表面附近，并使颗粒沿波导进行定向移 动 ${ }^{[49]}$. 2000 年 $\mathrm{NG}$ 等 $^{[50]}$ 发现半径为 $10 \mathrm{~nm}$ 的金纳 米颗粒可以被捕获在横向沟道波导上，并以最高 $4 \mathrm{~mm} / \mathrm{s}$ 的速度沿波导推进，其实验装置示意图如图 
4 (c). 颗粒在捕获后的移动速度可通过调节激光 功率、波导宽度及激光光束 $\mathrm{TM} / \mathrm{TE}$ 偏振态等进行 调控，如图 $4(\mathrm{~d})$. 纳米线波导及光子晶体波导等， 均可产生用于近场光镊捕获的修逝波，因此，极大 丰富了波导型光铌的设计和调控自由度.

近场修逝波光镊为二维平面内或片上微纳结构

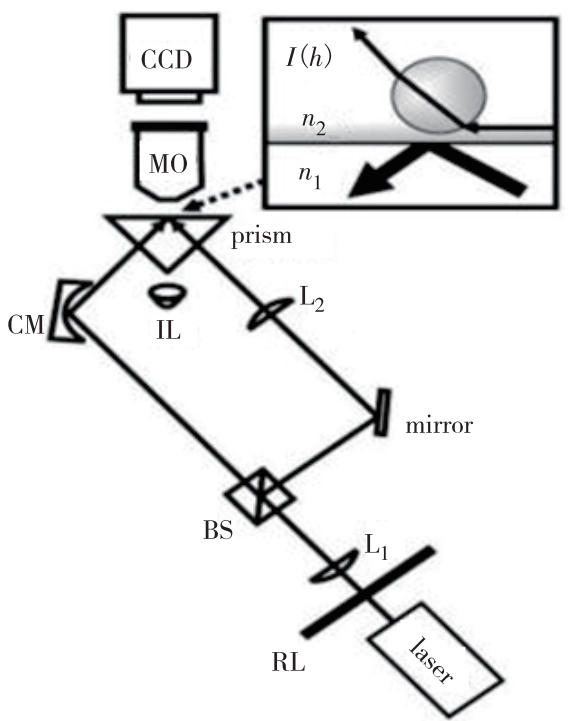

(a ) 棱镜全内反射近场光铌示意图 ${ }^{[45]}$

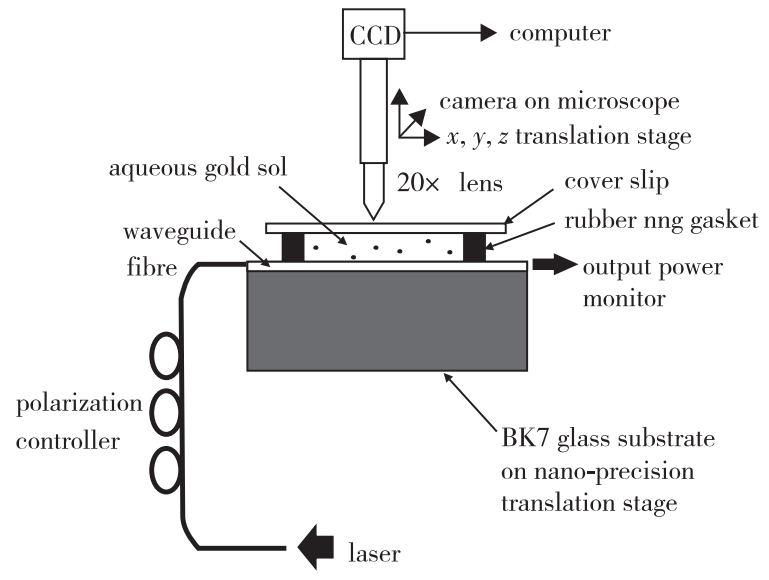

(c) 波导近场光镊示意图 ${ }^{[50}$
中的颗粒捕获和操控提供重要手段，在片上信号调 制、纳米结构搭建及颗粒传感芯片等方面具有重要 应用前景. 目前该技术对颗粒的操纵手段和动态调 控功能还不够丰富，需要进一步合理设计人射光源 参数和片上波导器件, 实现更加多样和动态化的片 上光镊操控效果.

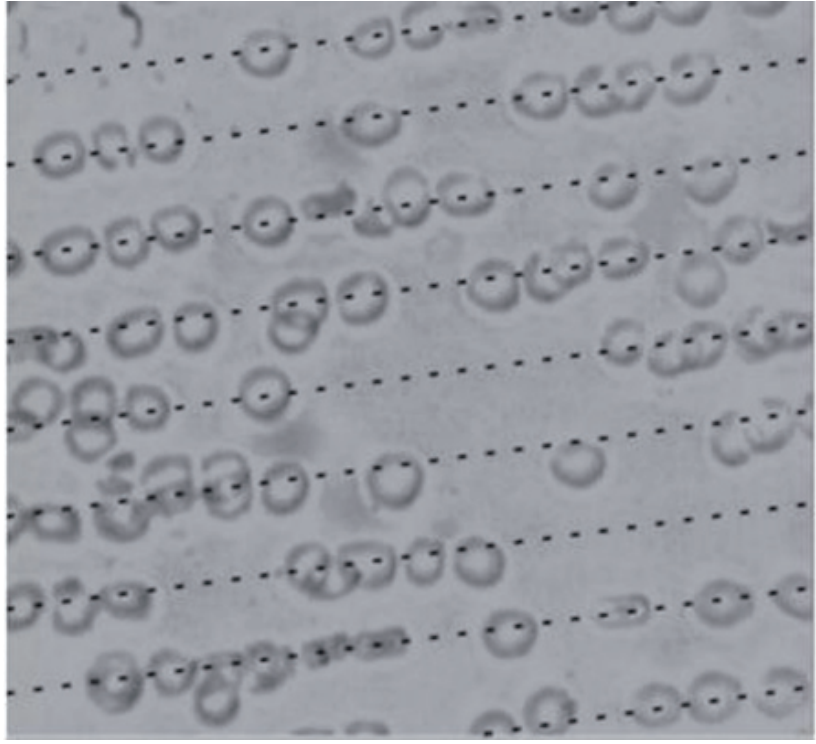

(b) 棱镜全内反射近场光镊捕获 $5 \mathrm{~mm}$ 聚苯乙烯微球 $[45$

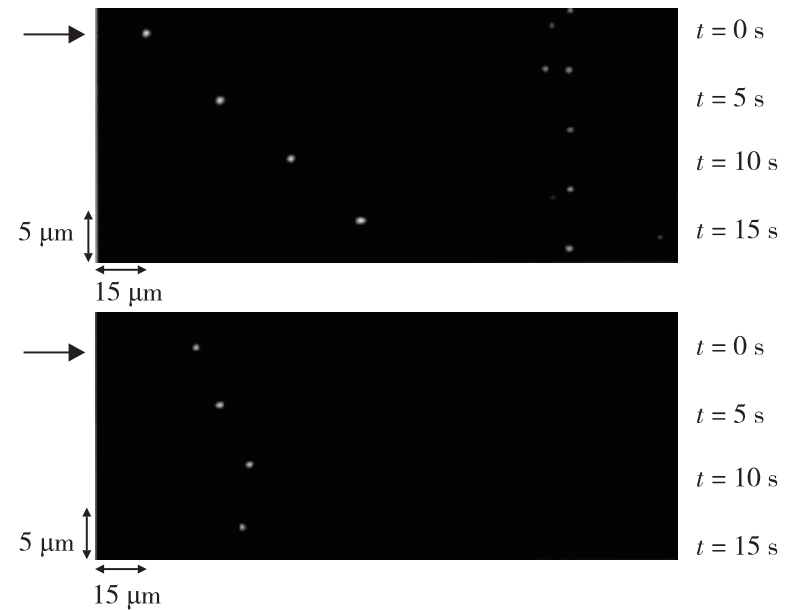

(d) 金纳米颗粒沿波导向前运动，上为TM偏振， 下为TE偏振，光从箭头指示的方向人射 ${ }^{[50]}$

图 4 近场修逝波光铌

Fig. 4 Near-field evanescent wave tweezers

\section{4 表面等离激元光铌}

表面等离激元（surface plasmon）是金属表面 自由电子与人射光子相互耦合与集体振荡产生的一 种仅在金属表面传播的电磁波模式，其强度沿远离 金属表面方向呈指数衰减. 与介质表面修逝波相 比，表面等离激元虽然也是一种修逝波，但其在金 属表面的近场局域性更强，具有更高的表面电磁场
增强因子和垂直方向电场梯度. 因此, 表面等离激 元光铌可以显著提高修逝场产生的光学梯度力，相 比近场修逝波光铌具有更强的捕获能力. 表面等离 激元还有突破传统光学衍射极限的能力，可以产生 纳米尺寸的电磁场焦点, 对于直接捕获生物分子等 纳米尺度样品具有独特优势。

按照激发方式不同，表面等离激元光镊可分为 
结构型和全光调控型. 由于不同的金属纳米结构可 以激发出不同的表面等离激元光场分布，通过设计 合适的金属纳米结构可将光场能量局限在远小于衍 射极限的区域内形成电磁场热点，提供极大的光学 梯度力，同时可以获得纳米尺度的颗粒捕获精度， 从而形成结构型表面等离激元光镊. 2007 年, RIGHINI 等 ${ }^{[51]}$ 最早提出表面等离激元光镊，就是基 于玻璃表面的金属圆盘阵列结构，在每个圆盘上激 发表面等离激元光场产生梯度力, 捕获溶液中的聚 苯乙烯微球排成特定图案，如图 5(a). 得益于聚 焦离子束刻蚀和电子束曝光等微纳加工技术的发 展，金属纳米结构中的表面等离激元光场特性得到 深人研究，推动了结构型表面等离激元光镊技术的 发展. 利用金属纳米天线结构、Bowtie 结构及金字 塔结构等，产生的多种不同功能的结构型表面等离 激元光镊，可应用于纳米颗粒分选和 DNA 检测等 领域 ${ }^{[52-54]}$.

结构型表面等离激元光镊虽然具有很高的捕获 精度，但对金属微纳结构的加工精度要求较高，且 固定结构无法对表面等离激元光场进行动态调控, 因此, 不能对捕获样品实现动态操控效果. 全光调 控型表面等离激元光镊为这个问题提供解决方 案 ${ }^{[26]}$. 全光调控型表面等离激元光镊利用高数值

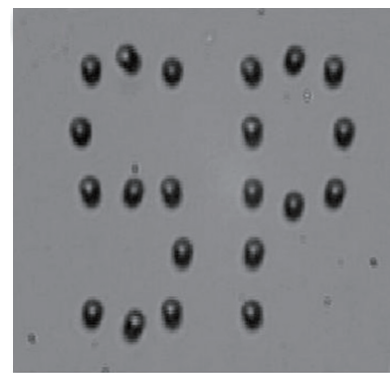

(a ) 结构型表面等离激元光铌 并行捕获 $4.8 \mu \mathrm{m}$ 聚苯乙烯微 球排成SP字母图案 ${ }^{[51}$

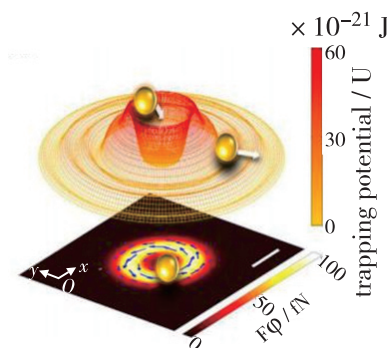

(c) 表面等离激元浴旋光镊对 金属颗粒的捕获和旋转 $[57$.

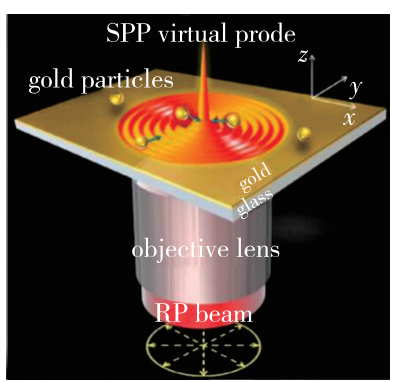

（b）聚焦表面等离激元 光铌捕获金属颗粒. ${ }^{[26]}$

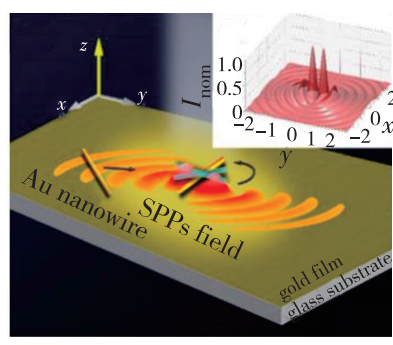

(d) 表面等离激元杂化模式对 单根金纳米线的捕获和旋转 操纵以及电场三维分布 ${ }^{[58}$
图 5 表面等离激元光镊

Fig. 5 Surface plasmon optical tweezers
孔径显微物镜将人射激光聚焦到单层纳米金属膜底 部，使其人射角范围内存在某一角度满足表面等离 激元共振激发条件，从而在金属膜上激发表面等离 激元并聚焦形成电磁场焦点，提供梯度力实现对颗 粒的捕获. 该光镊系统基于显微系统激发，在单层 金属膜上无需加工任何结构，可通过对人射光束的 相位、振幅及偏振态进行动态调控，实现表面等离 激元光场的动态操纵以及捕获颗粒的移动或旋转等 多种动态效果 ${ }^{[27,55-56]}$.

2013 年，闵长俊等 ${ }^{[26]}$ 首先提出并验证了全光 调控的聚焦表面等离激元光镊，实现对几十纳米到 几微米尺寸金属颗粒的稳定捕获，解决了传统光铌 难以捕获介观尺寸金属颗粒的难题，如图 5(b). 金属颗粒由于高散射和高吸收特性, 难以被普通激 光光镊捕获，但是聚焦表面等离激元光镊技术在捕 获和操纵金属颗粒方面具有明显优势，在表面增强 拉曼散射 (surface enhanced Raman scattering, SERS) 光谱、化学催化及微细加工方面具有重要应用前景.

在聚焦表面等离激元光镊研究基础上, 张聿全 等 ${ }^{[57]}$ 通过调控人射光的偏振和相位参量, 不仅可 以捕获纳米球、纳米线等多种颗粒样品，还能进一 步实现颗粒动态操控的效果. 研究将人射光相位变 为螺旋相位, 通过调节人射光的轨道角动量控制颗 粒旋转的速度和方向, 如图 5 (c). 研究还通过改 变人射光偏振态产生表面等离激元双焦点, 实现对 金属纳米线和半导体纳米线等样品的稳定捕获与旋 转角度操纵 ${ }^{[58]}$, 并应用于搭建金属膜上的纳米线 基结构, 如图 5 (d). 研究结果有望在光电功能芯 片和器件（如纳米逻辑门电路和纳米激光器）的构 建方面得到重要应用.

作为一种具有纳米量级捕获精度的光镊技术， 表面等离激元光铌可用于精确捕获与操纵 DNA 及 纳米颗粒等纳米尺寸样品. 表面等离激元的局域电 磁场增强效应可对所捕获样品的散射和光谱信号进 行放大，有利于样品识别和传感检测，可应用于生 物传感、分子检测及颗粒分选领域 ${ }^{[59]}$. 表面等离 激元光镊技术的进一步发展将为纳米光电子学、纳 米生物学和医药学的研究与发展带来深远影响. 目 前，该技术最大的难点在于金属的高吸收损耗特 性，不仅限制了表面等离激元的传播和光镊的操控 范围，产生的热效应也影响了颗粒捕获的稳定性. 如何克服这些缺点, 实现大范围和高精度的纳米颗 粒操纵, 将是该技术未来的重要研究内容. 


\section{5 光纤光铒}

光纤也可应用于光铌领域，具有结构简单、小 型化、可弯曲及成本低的优点. 由于光纤的模场较 小，使用过程中仅需将激光耦合到特制光纤内就能 形成光镊系统，摆脱了显微镜大体积聚焦系统的限 制, 在颗粒捕获操纵方面更灵活, 适用范围更广泛.

根据不同的光纤结构，光纤光铝可分为基于平 端面光纤的双光纤光镊、基于半球形自透镜端面的 双光纤或多光纤光镊、抛物线形单光纤光镊及大雉 角形单光纤光镊等 ${ }^{[60]}$. 这些结构型光纤可以通过 抛光 ${ }^{[61]}$ 、化学刻蚀 ${ }^{[62]}$ 、高分辨率微加工 ${ }^{[63]}$ 及热拉 伸 ${ }^{[64]}$ 等方法制造. 通过使用不同配置和结构的光 纤，可以实现高灵活性、高精度及高度一体化的光 镊操纵方式 ${ }^{[65]}$. 2008 年, YUAN 等 ${ }^{[66]}$ 利用双芯雉 形光纤光镊捕获并旋转椭圆形酵母细胞，通过改变 两路光束的功率比就可以控制酵母细菌的方向，图 6 (a) 为将酵母菌捕获在光纤端点的图像. 2016 年, $\mathrm{LI}$ 等 $^{[67]}$ 提出一种固定组装平行光纤阵列的方法, 可用于捕获和检测具有高通量、高分辨率及高选择 性的纳米颗粒和细胞. 实验具体方法基于固定直径 的光纤探针阵列, 利用装配技术将一组聚苯乙烯微 球固定在光纤探针表面形成微透镜阵列, 如图 6 (b)-(d). 由于微球对光的聚焦作用，该方法需 要的激光器功率比常规情况低 $1 \sim 2$ 个数量级, 同 时降低了热效应对光镊捕获和操纵的影响, 可实现

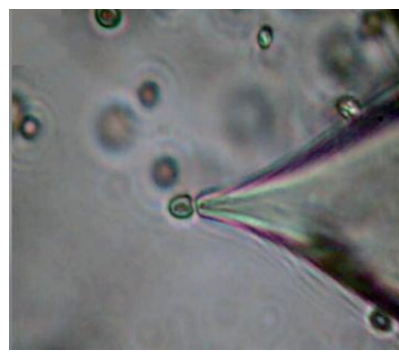

（a）双芯锥形光纤光铌捕获 椭圆形酵母细胞 $[66$

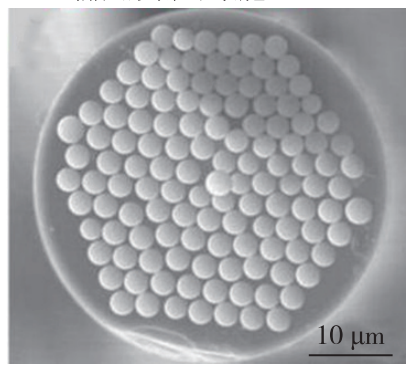

(c) 133 个微透镜的阵列 扫描电镜图像 ${ }^{[67}$

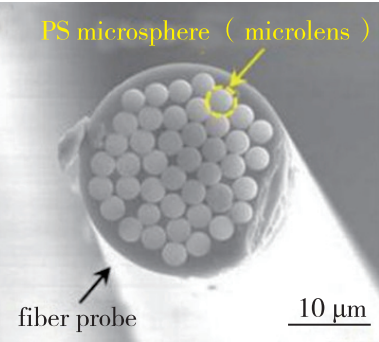

(b) 43 个微透镜的阵列扫描 电镜图像 ${ }^{[67]}$

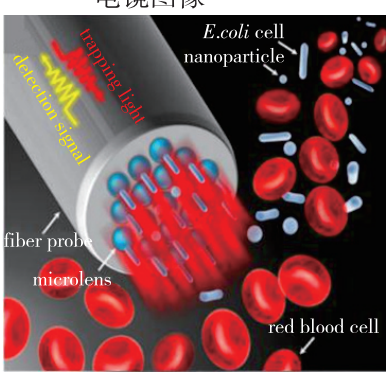

(d) 平行光纤阵列光镊 示意图 ${ }^{[67]}$
图 6 光纤光镊

Fig. 6 Optical fiber tweezers
微结构组装、加工、单细胞分析及光学分选等功能.

光纤光铌还可以通过在光纤端面加工微纳结 构，产生结构光场或表面等离激元，从而与结构光 束光镊和表面等离激元光镊结合 ${ }^{[68]}$, 实现更强大 的颗粒操纵功能. 目前光纤对出射光场的调控功能 有限, 除了产生焦点捕获颗粒, 其他动态操纵功能 还很少能实现, 所以需要进一步发展光纤出射光场 的调控技术，以推动光纤光镊技术的进步.

\section{6 热电光镊}

由于光热效应会引起溶液中的热对流和热扩散, 加大颗粒的布朗运动, 因此, 光热效应会阻碍光镊 捕获过程中的颗粒捕获. 但特殊条件下的光热效应 可对捕获过程有利，实验已经证明通过在介质中产 生能够将纳米颗粒移动到捕获位置的电热质流，有 助于光镊捕获，但在这种动态温度场中的颗粒仍会 频繁产生较大的位置波动 ${ }^{[69]}$. 因此, 基于光热效 应的高精度和高稳定性光镊系统仍难以实现.

2017 年 LIN 等 ${ }^{[70]}$ 提出一种新型热电光镊技术, 可通过光热效应稳定地捕获颗粒，其原理是通过金 膜的光热效应产生热梯度场，导致溶液中正负离子 移动，电荷作用共同提供热电场，最终通过电荷力 把带正电的颗粒稳定捕获在温度最高区域 ${ }^{[71]}$ ，如 图 7 (a)-(f). 该团队随后使用飞秒激光产生热电 光镊, 进一步提高了捕获的稳定性 ${ }^{[72]}$; 与光纤结 合开发光热电光纤光镊, 如图 $7(\mathrm{~g})$, 将热电光镊 推广到更多领域 ${ }^{[73]}$; 还对复杂体系中的热电光镊 原理进行详细的阐述 ${ }^{[74]}$, 如图 7 (h).

热电光镊由于需要纳米金属膜产生热梯度场, 最终颗粒捕获在金属膜表面，也属于二维光铝技 术. 相比修逝波光镊及表面等离激元光镊等二维光 铌技术，热电光镊因存在热电场，在二维平面内的 捕获范围可达半径 5 10 $\mu \mathrm{m}$ 的区域，同时在颗粒 捕获稳定性上具有更大优势, 其光吽刚度比普通光 镊高 $2 \sim 3$ 个数量级，在微纳结构组装和颗粒打印 领域具有重要应用前景. 热电光镊需要较低的人射 光功率 $($ 几 $\mathrm{mW})$, 若人射光功率太高, 反而会影 响颗粒捕获的稳定性，因此，无法用于纳米金属颗 粒局域场增强. 如何突破人射功率的限制, 仍需要 进一步研究探索.

\section{7 飞秒激光光镊}

飞秒激光具有超短脉冲宽度和超高瞬时功率的 特点, 利用飞秒激光作为光铌系统的光源可提供更 高的光学力, 降低光镊捕获所需的平均功率，同时 也可以明显增强所捕获样品的非线性光学效应，提 


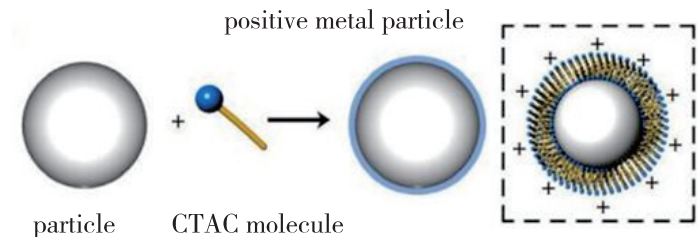

（a）通过阳离子表面活性剂（十六烷基三甲基氯化铵， CTAC ) 吸附对金属纳米颗粒进行表面电荷改性 ${ }^{[71]}$

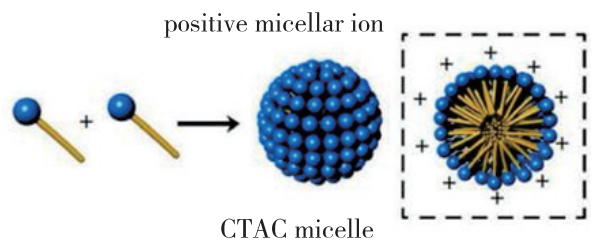

negative $\mathrm{Cl}$ ion

(b) CTAC胶束的形成 ${ }^{[71]}$

(c) 氯离子的示意图 ${ }^{[71]}$ $x / \mu \mathrm{m}$

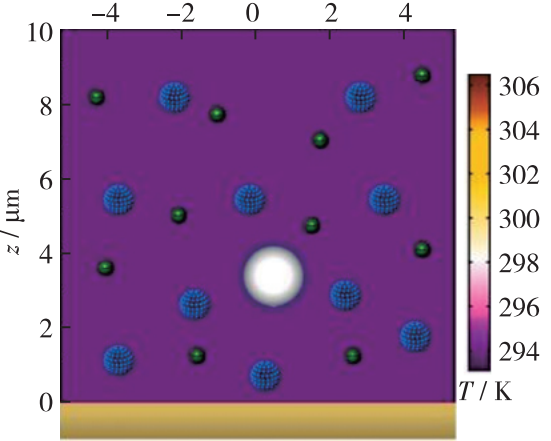

(d) 无光学加热时溶液中分散的 单个改性金属颗粒和离子 [71]

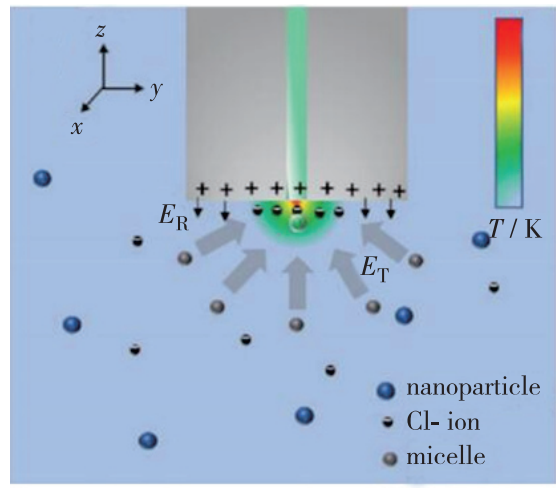

(g) 光热电光纤光铌 ${ }^{[73]}$ $x / \mu \mathrm{m}$

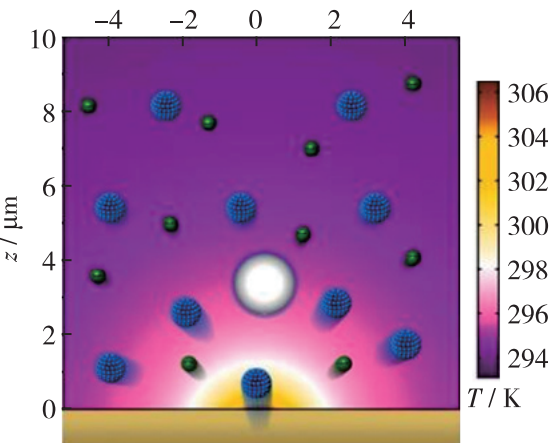

(e ) 光学加热下离子的热泳迁移 ${ }^{[71]}$

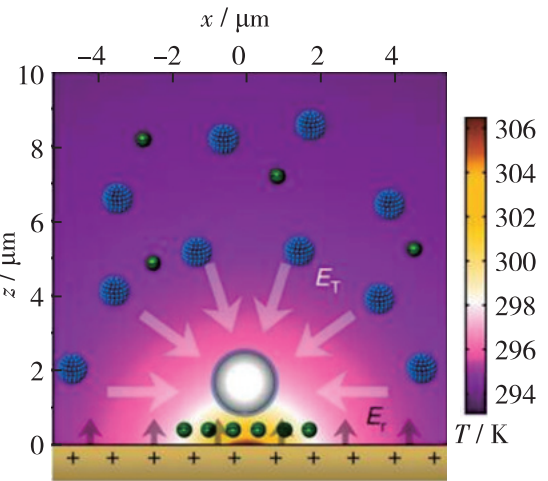

(f) 光学加热下的离子分布产生用于捕获 金属纳米颗粒的热电场 $E_{\mathrm{T}}$, 排斥电场 $E_{\mathrm{r}}$ 源于基底对 $E_{\mathrm{T}}$ 产生的感应电场 ${ }^{[71]}$

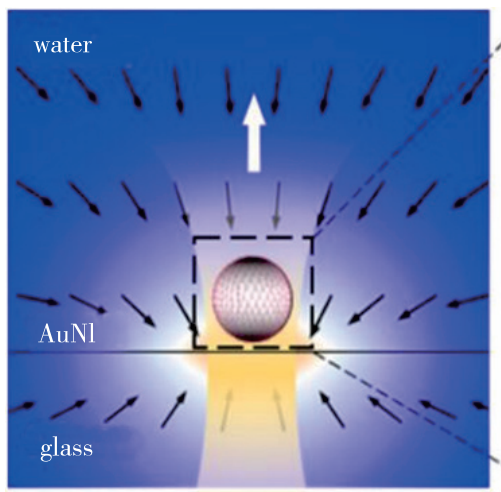

(h) 复杂体系中单个颗粒的热电场模拟 ${ }^{[74]}$

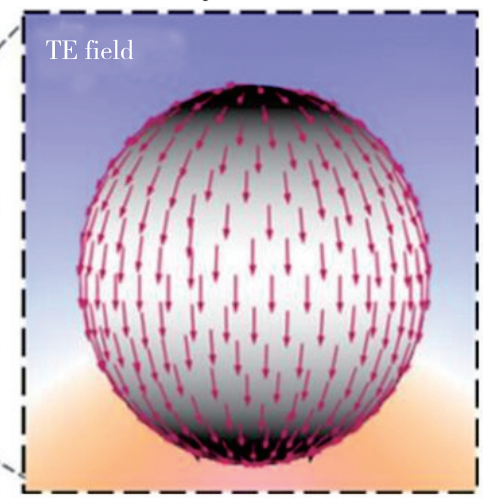

图 7 热电光铌

Fig. 7 Opto-thermoelectric tweezers

升光镊技术的多样性 ${ }^{[75]}$.

2012 年，ROXWORTHY 等 ${ }^{[76]}$ 在蝶形金属纳米 天线阵列的表面等离激元光镊基础上, 采用飞秒激 光作为输人光源来增强瑞利和米氏颗粒的捕获，与 连续激光光镊相比, 光陎刚度提升 2 倍. 2015 年, SHAKHOV 等 ${ }^{[77]}$ 提出一种使用飞秒光镊捕获介质微 球进行纳米图案刻写的新方法, 通过使用捕获的微 球作为微透镜将光束汇聚, 在短时间 (约 $45 \mu \mathrm{s}$ ) 内产生较大光学力 (最高达 $0.1 \mathrm{nN})$, 以克服颗粒 与基底表面之间的黏合作用，最终达到 $130 \mathrm{~nm}$ 的 刻写精度.

在飞秒脉冲高峰值功率作用下，某些颗粒样品
（如金属纳米颗粒）的非线性光学效应得到很大增 强, 出现一些特殊的光镊捕获结果 ${ }^{[78-79]} .2010$ 年, 降雨强等 ${ }^{[80]}$ 报道线偏振飞秒激光捕获金纳米颗粒 时出现的光阱䢃裂现象，即在飞秒激光与金纳米颗 粒的非线性作用下，连续激光下只能捕获单个颗粒 的光学势阱会一分为二, 同时捕获两个颗粒. 在此 基础上, 2018 年, 张聿全等 ${ }^{[81]}$ 进一步研究飞秒矢 量光束聚焦光场对金属纳米颗粒的非线性力学效 应，并利用单个矢量偏振飞秒光束产生多个非线性 光学势阱，实现对金纳米颗粒的多重稳定捕获，如 图 8. 结果表明, 对于电场强度敏感的可极化纳米 结构样品（如金属、半导体结构及原子等）, 其非 
线性捕获结果可通过改变人射光的偏振状态和强度 进行灵活调控. 该工作揭示并验证了线性与非线性 条件下，纳米颗粒在光场中受力的物理机制，为光 镊技术研究由线性光学跨入非线性光学领域, 产生
更多非线性光学操控技术奠定基础. 由于非线性光 学效应的复杂性，飞秒光铌中光与物质非线性相互 作用的一些物理机制仍不明确，有待进一步探索.

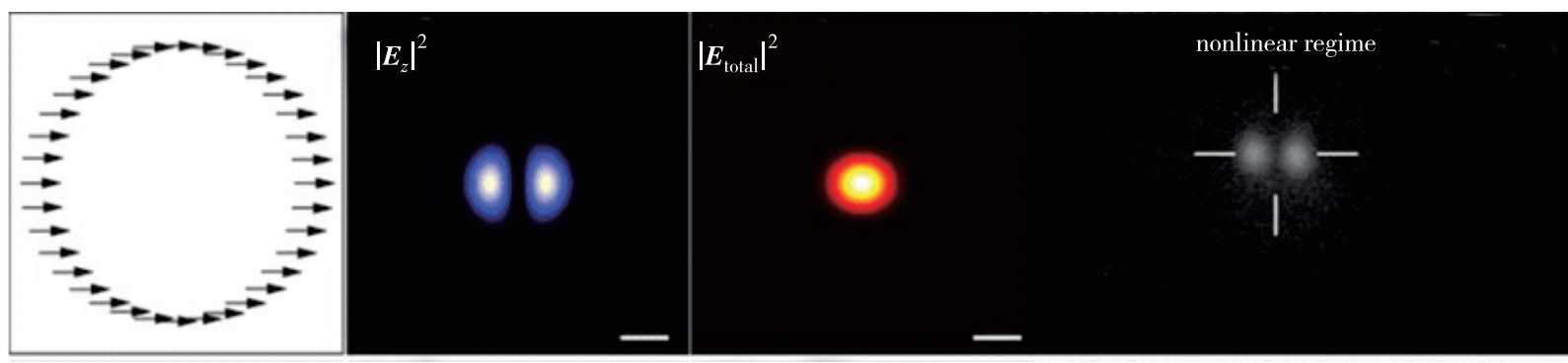

( a ) 矢量偏振阶数 $m=0$ 的柱矢量光束截面的偏振分布、纵向电场分量强度 $\left|\boldsymbol{E}_{z}\right|^{2}$ 、总电场强度 $\left|\boldsymbol{E}_{\text {total }}\right|^{2}$ 和 对应矢量偏振飞秒脉冲激光在非线性状态下捕获 $60 \mathrm{~nm}$ 金颗粒的结果

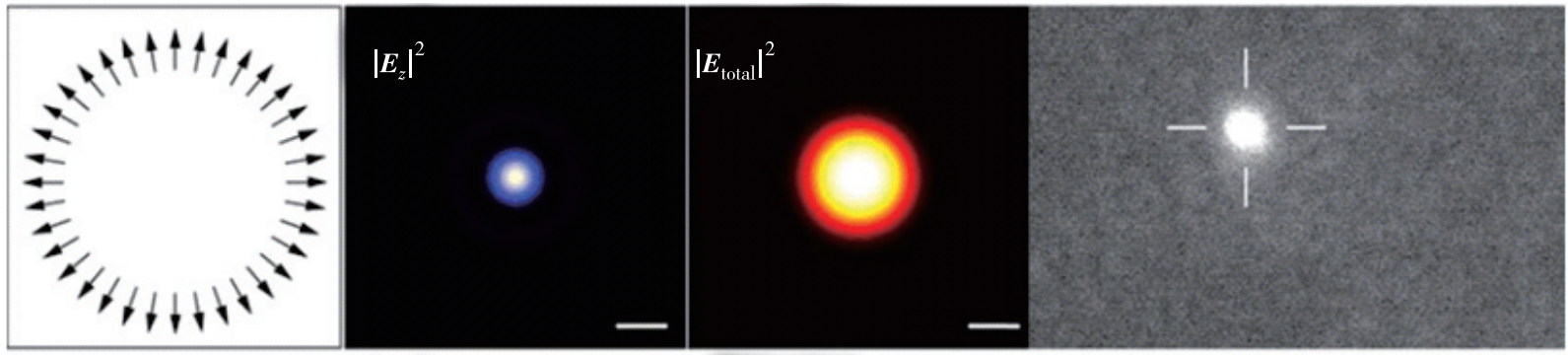

(b) 矢量偏振阶数 $m=1$ 的柱矢量光束截面的偏振分布、纵向电场分量强度 $\left|\boldsymbol{E}_{z}\right|^{2}$ 、总电场强度 $\left|\boldsymbol{E}_{\text {total }}\right|^{2}$ 和 对应矢量偏振飞秒脉冲激光在非线性状态下捕获 $60 \mathrm{~nm}$ 金颗粒的结果

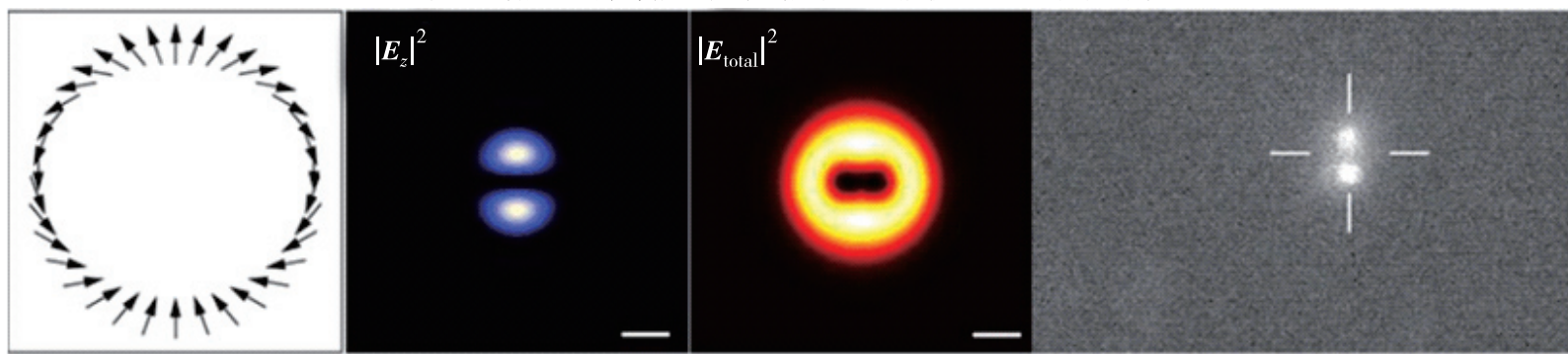

( c ) 矢量偏振阶数 $m=2$ 的柱矢量光束截面的偏振分布、纵向电场分量强度 $\left|\boldsymbol{E}_{z}\right|^{2}$ 、总电场强度 $\left|\boldsymbol{E}_{\mathrm{total}}\right|^{2}$ 和 对应矢量偏振飞秒脉冲激光在非线性状态下捕获 $60 \mathrm{~nm}$ 金颗粒的结果

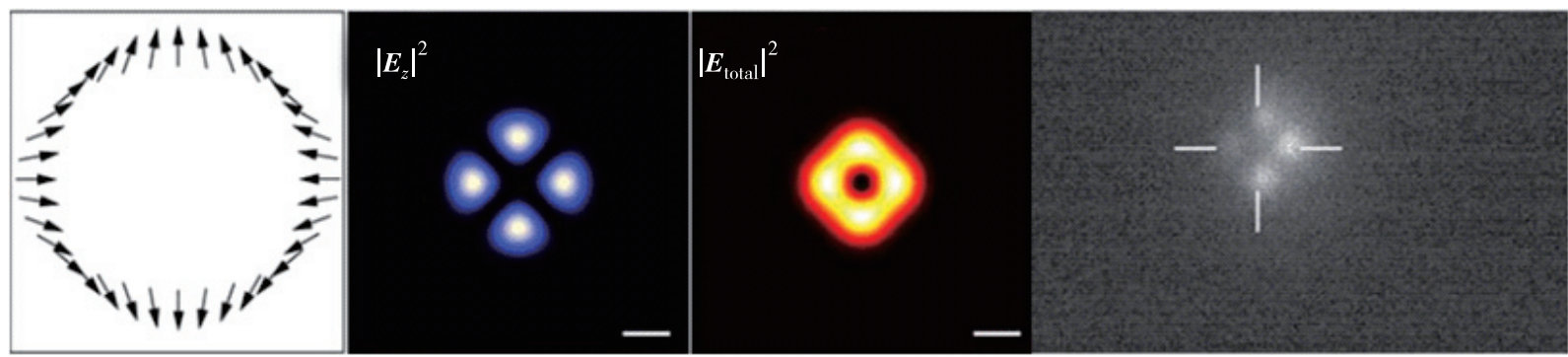

(d) 矢量偏振阶数 $m=-1$ 的柱矢量光束截面的偏振分布、纵向电场分量强度 $\left|\boldsymbol{E}_{z}\right|^{2}$ 、总电场强度 $\left|\boldsymbol{E}_{\mathrm{total}}\right|^{2}$ 和 对应矢量偏振飞秒脉冲激光在非线性状态下捕获 $60 \mathrm{~nm}$ 金颗粒的结果

图 8 金纳米颗粒在多种飞秒矢量光束聚焦条件下的非线性捕获 $\left.{ }^{[81}\right]$

Fig. 8 Nonlinear trap of gold nanoparticles under various femtosecond vector beam focusing conditions ${ }^{[81]}$

\section{8 异常光学力光镊}

近年来部分研究集中在由特殊光学效应引起 的、与传统光铝中颗粒受力不同的异常光学力效应
及相关光铝技术上，这里统称为异常光学力光镊， 如光学拉力、负折射光学力及自旋-轨道耦合横向 光学力光镊等. 
通常大尺寸不透明颗粒（如金属颗粒）主要受 到沿人射光传播方向的辐射压力作用进行移动. 通 过调节周围颗粒对目标颗粒的散射，使目标颗粒的 受力与光传播方向相反, 或通过设计光束使目标颗 粒前向散射强度大于后向散射，从而产生反方向的 散射力, 如图 9 (a) 和图 $9(\text { b })^{[82]}$. 在这些情况下 颗粒不会被推走，而是会被拉向光源方向，所以这 类颗粒受力被称为光学拉力 ${ }^{[82-83]}$.

在负折射材料中折射光束会沿负方向传播, 所 产生的光学力也会沿负折射的方向. 当激光在水和 金属光子晶体结构界面处发生折射时，可在结构中 激发表面等离激元布洛赫模式产生负折射效果，使 光沿负折射方向传播, 并推动颗粒沿负折射方向移 动, 如图 $9(\mathrm{c})^{[84]}$.

自旋-轨道耦合效应产生的横向光学力如图 9 (d) ${ }^{[85]}$. 当人射光为左旋圆偏振光带有自旋角动 量，金属表面的颗粒会因为散射而产生光子自旋角 动量到轨道角动量的转化, 根据角动量守恒定律, 在金属表面产生向左侧横向传播的表面等离激元 波，从而对颗粒产生反方向（指向右侧）的横向作 用力. 虽然此时人射光和颗粒均为圆对称的，但是 金属表面的存在破坏了整个系统的对称性，使自 旋-轨道耦合仅能产生单向传播的表面波，从而对 颗粒产生横行的反冲力. 该效应对介质波导表面的 颗粒同样有效，可通过自旋-轨道耦合在介质波导 内产生单向传播的波导模式，并对颗粒产生反方向 作用力. 由于光子自旋-轨道耦合需要满足动量守 恒, 因此, 可通过改变人射光的偏振手性来调控所

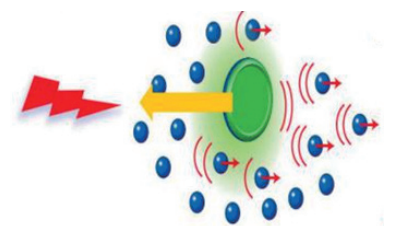

（a）由其他颗粒散射产生的 光学拉力光铝 ${ }^{[82]}$

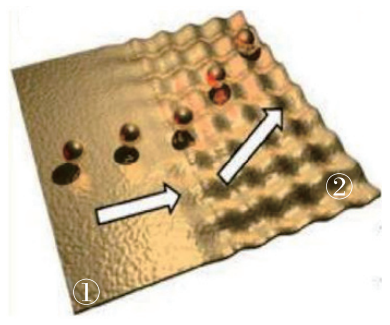

(c) 负折射光镊 ${ }^{[84]}$

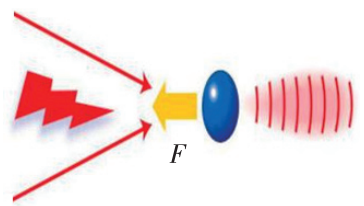

(b) 由颗粒自身散射产生的 光学拉力光铌 ${ }^{[82]}$

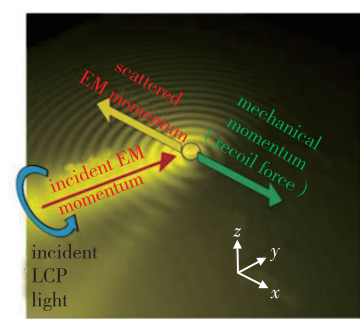

(d) 自旋-轨道耦合产生 横向光学力光铌 $[85]$
图 9 异常光学力光铌

Fig. 9 Extraordinary optical forces and tweezers
产生表面波或波导模式的传播方向、以及颗粒所受 横向力的方向 ${ }^{[55-87]}$. 这种横向力的产生不需要光束 聚焦，因此，可以作用于大范围内的所有颗粒，在 手性颗粒分选等领域有重要应用前景.

异常光学力的研究丰富了光镊的操控手段, 也 加深对新颖光学效应背后物理机制的理解. 目前对 异常光学力光镊的研究还处于物理探索方面, 如何 基于异常光学力产生新应用，还需要进一步深人 研究.

\section{3 光镊技术的应用}

光铌技术在科学研究及生产生活等多个领域已 得到重要应用. 以下介绍光铌技术代表性的应用方 向，包括生物细胞及大分子操纵、表面增强拉曼光 谱检测、光铌打印、光铌三维显示及光铌驱动微型 发动机.

\section{1 生物细胞及大分子操纵}

生物医学领域是光镊技术最重要的应用方向之 一. 由于光铌可捕获和操控的样品尺寸在纳米到微 米量级，与生物大分子和细胞的尺寸对应，光铝已 成为生物分子和细胞学研究领域的重要工具.

在细胞研究方面, 红细胞作为氧气载体在生命 活动中起着至关重要的作用, 而红细胞聚集会影响 血液的流变特性，导致严重的血管问题. 2013 年, ZHONG 等 ${ }^{[88]}$ 提出使用光铌捕获和操纵活小鼠皮下 毛细血管内的红细胞, 可用来清除堵塞的微血管, 实现非接触式微操作. 实验通过光镊捕获阻塞毛细 管的红细胞，当捕获力大于摩擦力时，血液在毛细 血管中恢复流动，使毛细血管重新充满新鲜血液. 该研究成果表明光镊技术有望应用于血栓等疾病的 治疗.

光镊技术不仅可以操纵细胞 ${ }^{[89]}$, 还可操纵 DNA 等生物大分子. 由于分子尺寸太小，目前绝大 部分光镊技术不足以直接稳定地捕获生物分子，但 可以通过一些微颗粒间接操纵分子，例如可以与生 物大分子结合的微米尺寸聚苯乙烯微球. 因此，在 大多数单分子光镊实验中，这些微球被当做生物大 分子的手柄系统，在样品室内被光镊捕获并进行操 作. 1997 年，WANG 等 ${ }^{[90]}$ 将单个 DNA 分子的一端 通过 RNA 聚合酶复合物固定在盖玻片表面，另一 端连接一个微球; 再用光镊将微球捕获并固定在一 个光吽中，通过使用压电驱动平台将盖玻片相对于 微球移动来拉伸 DNA，从而实现检测 DNA 性质. 
2011 年, PANG 等 ${ }^{[91]}$ 利用光镊稳定捕获了蛋白质分 子，并且将蛋白质展开，研究成果有望发展成为可
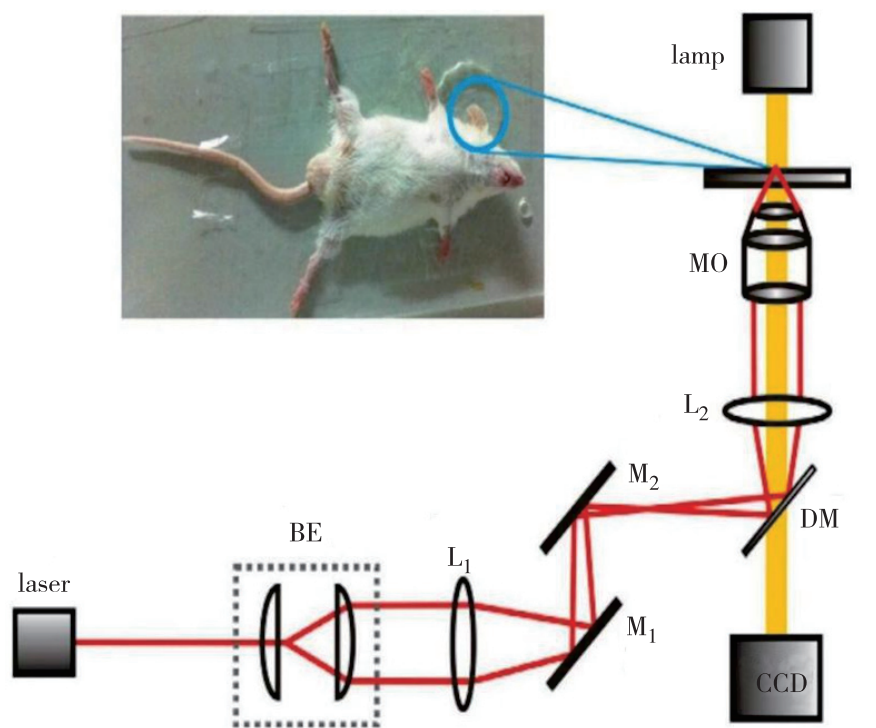

（a ）使用红外光铌捕获和操纵活小鼠皮下毛细血管内的 红细胞实验装置示意图

用于单个蛋白质检测的生物传感器，在药物研发及 疾病和感染检测中具有广阔应用前景.

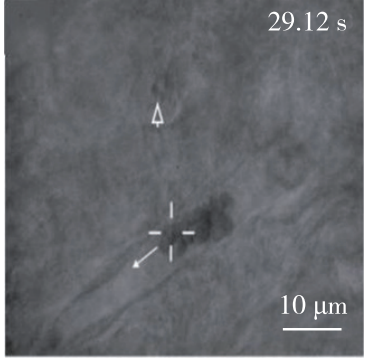

(b) 捕获堵塞在微血管中的 红细胞

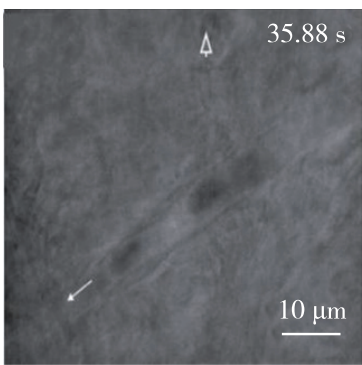

（d）红细胞的移动距离约为 $12 \mu \mathrm{m}$ 当光镊关闭时, 毛细血管中的 其他静止红细胞开始移动

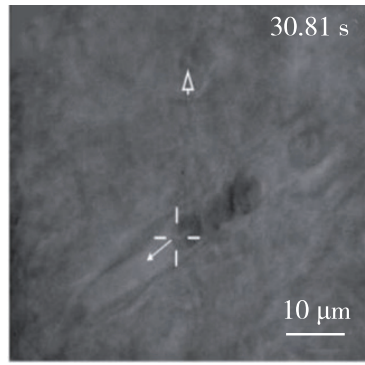

(c) 被捕获的红细胞沿着 毛细管中光陎移动

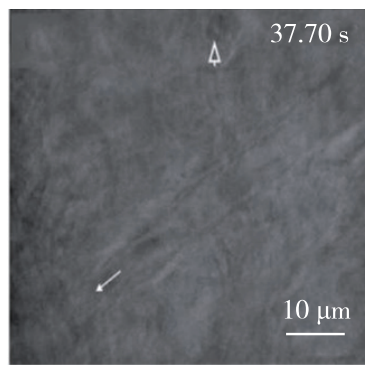

（e）血流恢复正常流量

图 10 光镊疏通血管 ${ }^{[88}$

Fig. 10 Optical tweezers dredge blood vessels ${ }^{[88]}$

\section{2 表面增强拉曼光谱检测}

当一定频率的激光照射到样品上时，由于不同 样品中分子化学键的振动频率不同，使散射光频率 与人射光不同，且散射光频率分布具有唯一性，其 光谱即为拉曼光谱. 拉曼光谱可用来检测和鉴别分 子的种类，也被称为分子的指纹谱. 但是，拉曼散 射信号强度仅为传统瑞利散射的千分之一，难以检 测到. SERS 技术利用金属纳米颗粒的局域电磁场 增强特性增强样品分子的拉曼信号, 通过在样品分 子溶液中直接加人大量金属纳米颗粒，之后使用人 射光照射金属颗粒，通过颗粒周围的局域电场增强 拉曼信号. 但由于金属纳米颗粒布朗运动的随机 性，拉曼增强信号存在强度不稳定、重复性差和不 可控等缺点. 而金属纳米颗粒可以被表面等离激元 光镊技术捕获和操控，因此，可以利用光镊技术发 展出信号稳定且可动态操控的表面增强拉曼光谱检 测技术 ${ }^{[92]}$.

2013 年, 沈军峰等 ${ }^{[93]}$ 首次实现基于表面等离 激元光镊动态操控的 SERS 技术. 研究通过聚焦表 面等离激元光镊捕获了单个直径为 $250 \mathrm{~nm}$ 的金纳
米颗粒，并增强颗粒附近电磁场，实现 Rh6G 分子 的动态可控 SERS 检测，拉曼信号增强因子可达到 $5.67 \times 10^{9}$. 其原理是在紧聚焦条件下，利用径向 偏振光人射激发表面等离激元焦点，并与被捕获的 金属颗粒之间产生很强的电磁场耦合作用，使金属 颗粒与金属膜之间的间隙内产生超高电磁场增强效 应，从而使间隙区域内的分子能够被激发出更强的 拉曼信号 ${ }^{[94-95]}$. 2017 年，张聿全等 ${ }^{[96]}$ 利用双束表 面等离激元光铌技术，实现单个半径为 $100 \mathrm{~nm}$ 金 颗粒的稳定捕获和周围颗粒的排斥，应用于可动态 调控的单分子拉曼增强检测. 由于光镊可以捕获金 属颗粒并移动到指定位置，该技术可用于金属膜表 面不同位置分子的拉曼信号增强探测，甚至可以使 颗粒来回扫描获得二维平面上所有分子的高分辨率 拉曼扫描成像，对于 SERS 光谱检测技术发展具有 重要意义.

\section{3 光镊打印}

光镊打印是光镊技术的一个重要应用方向，其 原理是通过光铌把微纳米颗粒捕获并固化于特定位 置，从而在基底上打印出由微纳米颗粒组成的图 
案, 获得具有一定功能的微纳结构 ${ }^{[97]}$. 光镊打印 的步骤通常是先通过光镊捕获和操纵单个颗粒, 将 颗粒移动到设计好的位置; 基于光热效应使颗粒固 定在基板上; 不断重复这个过程, 最终获得设定的 微纳结构 ${ }^{[98-99]}$.

2013 年, DO 等 ${ }^{[100]}$ 通过使用 2 种不同波长的 激光控制, 实现金纳米棒的沉积和结构打印. 研究 首先利用一束与金纳米棒共振波长不同的激光，实 现稳定光镊捕获, 并移动单根金纳米线到预定位 置; 再利用另一束激光激发金纳米棒表面等离激元 共振产生光热效应, 实现纳米棒的沉积固化并形成 结构. 2016 年，LIN 等 ${ }^{[101]}$ 提出一种气泡光镊打印 技术. 研究将激光聚焦在金属膜的基板上，利用金 属膜的光热效应在胶体悬浮液和金属膜的界面处产 生微气泡. 微气泡通过马兰哥尼对流、表面张力、 气泡压力和黏附力的共同作用, 将其附近的胶体颗 粒捕获并固定在基板上. 通过移动激光焦点位置来 移动微气泡, 就可以沿移动轨迹把聚苯乙烯微球颗 粒稳定打印在基板上，得到设定图案.

光镊打印技术拓展了光镊在微加工领域的应 用，具有系统简单和成本较低的优势，但其在打印 精度、打印速度及颗粒材料选择等方面还有待进一 步的深人研究.

\section{4 光镊三维显示}

三维空间显示技术在日常生产和生活方面应用 广泛, 其实现需要 2 个条件: (1) 通过光学全息的方 法在空间产生一个三维光场; (2) 通过散射介质 (如毛玻璃和雾屏系统), 将三维光场散射至各个方 向, 便于直接观察三维光场分布. 近年来, 研究发 现光镊技术也可用于自由空间三维显示，能够在稀 薄空气中产生三维光学图像, 这些图像几乎从任何 方向都是可见的.

2018 年, SMALLEY 等 ${ }^{[102]}$ 提出一种光镊捕获 散射颗粒在自由空间的三维显示方法，产生的全彩 色三维图像具有色域大、细节细淢及表面斑点低的 优点, 引起广泛研究关注. 其工作原理是首先在近 乎不可见 $(\lambda=405 \mathrm{~nm})$ 的光吽中由斜像散和球差 结合形成聚焦光镊捕获点, 在自由空间捕获一个微 米级的不透明颗粒. 所捕获的颗粒被光镊移动到用 于显示的空间区域内，再被光镊操控来回快速扫 描. 通过共线 RGB 激光系统产生全彩色三维光场 图案，照射到快速扫描的颗粒区域，由于颗粒对光 场沿各方向的散射及颗粒快速扫描形成的人眼视觉 暂留效应，裸眼可以直接观察到空间中的全彩色低
散斑三维图像, 如图 $11^{[102]}$.

光镊三维显示有望与全息光镊技术结合, 实现 多样的立体化成像与操控效果, 在裸眼三维显示及 人机交互领域具有重要发展潜力.

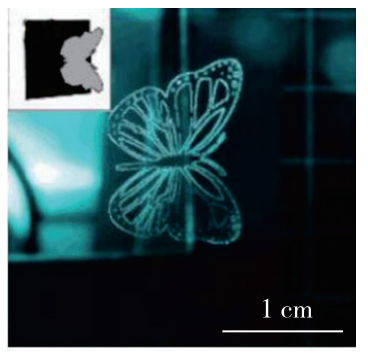

(a ) 蝴蝶图像

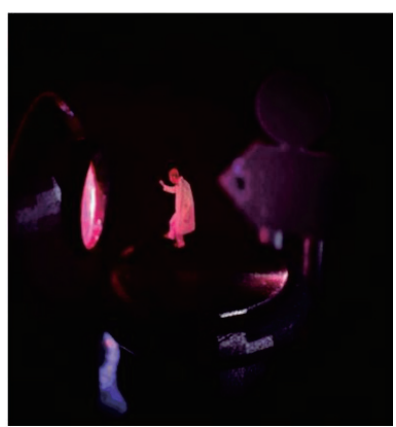

（c）成像点的几何形貌

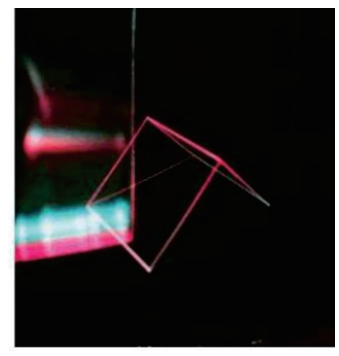

(b) 三维棱镜

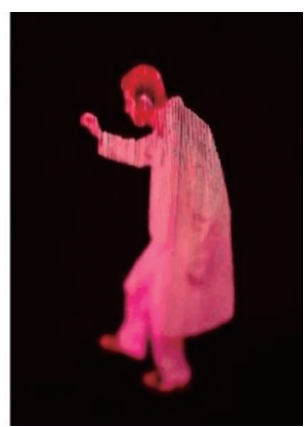

(d) 投影图像的特写
图 11 光镊三维显示 ${ }^{[102]}$

Fig. 11 Optical tweezers for 3D display ${ }^{[102]}$

\section{5 光镊驱动微型发动机}

近年来针对热机的小型化需求，探索新的驱动 方式为微型机械设备提供动力成为研究热点 ${ }^{[103-104]}$. 在微型热机运行过程中, 外部环境的改变会显著影 响微型热机的运行. 因此, 可以通过光铌产生的力 学和热学效应, 操控微纳米颗粒样品形成微型热机 运动.

2014 年, QUINTO-SU ${ }^{[105]}$ 指出在发动机动力学 受布朗运动控制的环境中，基于光铝技术，微米尺 寸的热机可以在两个热水域 (温度分别为 $22{ }^{\circ} \mathrm{C}$ 和 $90{ }^{\circ} \mathrm{C}$ ) 之间使用经典斯特林循环，每个循环所提 取功的平均值为 $4.5 \times 10^{-22} \mathrm{~J}$. 一个完整周期由颗 粒从光束的几何焦点或束腰以下开始，此时焦点处 溶液温度低于在大气压 $P_{0}$ 下的饱和温度. 颗粒被 光的梯度力和散射力共同作用并推向焦点. 当颗粒 接近几何焦点时, 光强度逐渐增加, 颗粒的表面温 度和捕获梯度力相应提高, 如图 12 中过程 $1-2$. 在靠近俘获光束焦点位置时, 颗粒表面温度升高较 大，且颗粒的移速足够快，与颗粒顶部表面接触的 液体突然过热（图 12 中过程 2-3), 在大气压 $P_{0}$ 
状态下达到临界温度。过热的液体通过形成气泡而 释放热量，气泡随后在周围液体的静水压力下破 裂, 此时微球经由气泡膨胀驱动回到起始位置（图 12 中过程 3-4-1），循环重新开始.

此外, 光镊可以操纵颗粒做机械运动, 如光学
旋涡光镊可以作为光学扳手让微型机械结构快速旋 转，用于液体混合搅拌、微流泵等领域. 光镊技术 还可以在微观条件下控制力学和热学效应, 在微型 机械领域具有广泛应用.

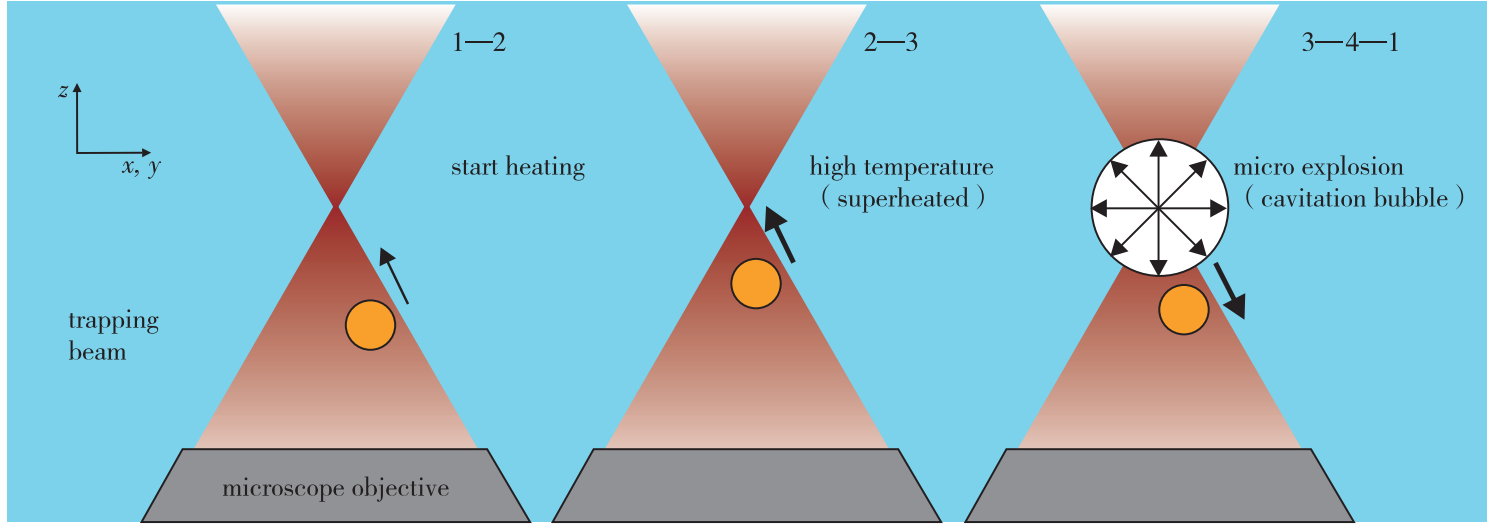

图 12 光镊热机中颗粒运动轨迹 ${ }^{[105]}$

Fig. 12 Microparticle trajectory in the optical tweezer ${ }^{[105]}$

\section{4 总结与展望}

本文对光镊技术的基本原理、代表性光镊技术 及其特色应用的相关研究进展进行系统综述, 分析 认为近 10 年来光铝技术的发展受到新光场、新结 构及新效应的大力推动. (1) 新光场表现在三维捕 获所用激光从传统单束激光向全息光场/结构光束/ 飞秒脉冲激光发展，二维捕获光场由介质表面修逝 波向金属表面等离激元发展，这些新型光场的加人 为光镊捕获与操控的精度、范围及自由度带来显著 提升; (2) 新结构表现在光纤微球阵列、金属纳米 天线及结构型探针针尖等微纳米尺寸光学新结构的 引人, 增强了纳米尺寸样品操纵的精确性和稳定 性; (3) 新效应包括引起广泛关注的光子自旋-轨道 耦合效应、负折射效应及光热电效应等，产生新的 光铌原理, 为光镊技术带来多样化的操控手段. 在 这些新技术的发展和推动下, 光铌已在生物细胞、 分子操纵、微纳结构打印及三维显示等领域展开广 泛应用.

研究认为光镊技术未来的发展趋势表现在：(1) 更小的捕获样品尺度. 以表面等离激元光镊为代 表，通过纳米金属结构产生几纳米尺寸的光学焦 点, 可以直接捕获和操控单个 DNA 分子样品, 应 用于单分子光谱检测; (2) 对样品物理或化学性质 更强大地操纵. 以飞秒激光光镊为代表，其不仅可
以捕获和操纵颗粒, 还可以进一步改变颗粒的光学 非线性性质，得到不同于线性条件下的结果; (3) 更多样化的操控手段. 以结构光束光铌和异常光学 力光镊为代表, 将更多样化的光束、结构和光学效 应用于光铌操控, 产生新颖的操控效果; (4) 更广 泛的应用范围. 以光镊打印和光镊三维显示等新应 用为代表，通过光镊强大的操控能力，寻找光铌与 其他研究领域的新结合点, 拓展光镊的应用范围.

光镊技术的研究不仅增进对光自身性质以及光 与物质相互作用的理解, 也增强了人类对光的驾驭 能力及对微观样品的操控能力, 推动光学与其他学 科的交叉结合，促进大量创新应用的出现. 未来光 镊技术必将迎来更加蓬勃的发展.

基金项目: 国家自然科学基金资助项目（91750205，61935013, 61975128)

作者简介：闵长俊 $(1981-)$, 深圳大学特聘教授、博士生导师. 研究方向: 微纳光学. E-mail: cjmin@ szu.edu.cn 袁运琪 (1994-) , 深圳大学硕士研究生. 研究方向 光镊技术. E-mail: 1810285003@ email.szu.edu.cn 闵长俊、袁运琪为共同第一作者.

引文: 闵长俊, 袁运琪, 张聿全, 等. 操纵微纳颗粒的 “光之 手”一光镊技术研究进展 $[\mathrm{J}]$. 深圳大学学报理工版, $2020,37(5): 441-458$

\section{参考文献 / References:}

[ 1 ] ASHKIN A, DZIEDZIC J M, BJORKHOLM J E, et al. Observation of a single-beam gradient force optical trap for dielectric particles $[\mathrm{J}]$. Optics Letters, 1986, $11(5)$ : 
288-290.

[ 2 ] RAMSER K, HANSTORP D. Optical manipulation for single-cell studies $[\mathrm{J}]$. Journal of Biophotonics, 2010, 3 (4) : 187-206.

[ 3 ] LI T C, KHEIFETS S, RAIZEN M G. Millikelvin cooling of an optically trapped microsphere in vacuum $[\mathrm{J}]$. Nature Physics, 2011, 7(7) : 527-530.

[ 4 ] JONAS A, ZEMANEK P. Light at work: the use of optical forces for particle manipulation, sorting, and analysis [J]. Electrophoresis, 2008, 29(24) : 4813-4851.

[ 5 ] QUIDANT R, GIRARD C. Surface-plasmon-based optical manipulation $[\mathrm{J}]$. Laser \& Photonics Reviews, 2008, 2 $(1 / 2): 47-57$.

[ 6 ] LUKOSZ W, KUNZ R E. Light-emission by magnetic and electric dipoles close to a plane interface. I. total radiated power $[\mathrm{J}]$. Journal of the Optical Society of America, 1977, $67(12)$ : 1607-1615.

[ 7 ] GUSSGARD R, LINDMO T, BREVIK I. Calculation of the trapping force in a strongly focused laser-beam $[\mathrm{J}]$. Journal of the Optical Society of America B: Optical Physics, 1992, 9(10): 1922-1930.

[ 8 ] CHAUMET P C, NIETO-VESPERINAS M. Time-averaged total force on a dipolar sphere in an electromagnetic field [J]. Optics Letters, 2000, 25(15) : 1065-1067.

[ 9 ] HARADA Y, ASAKURA T. Radiation forces on a dielectric sphere in the Rayleigh scattering regime $[\mathrm{J}]$. Optics Communications, 1996, 124(5/6) : 529-541.

[10] GREHAN G, MAHEU B, GOUESBET G. Scattering of laser-beams by mie scatter centers-numerical results using a localized approximation [J]. Applied Optics, 1986, 25 (19) : 3539-3548.

[11] CHAUMET P C, NIETO-VESPERINAS M. Coupled dipole method determination of the electromagnetic force on a particle over a flat dielectric substrate $[\mathrm{J}]$. Physical Review B, 2000, 61(20): 14119-14127.

[12] LOCK J A. Calculation of the radiation trapping force for laser tweezers by use of generalized Lorenz-mie theory. I. localized model description of an on-axis tightly focused laser beam with spherical aberration [ J ]. Applied Optics, 2004, 43(12) : 2532-2544.

[13] SONG Y G, HAN B M, CHANG S. Force of surface plasmon-coupled evanescent fields on mie particles $[\mathrm{J}]$. Optics Communications, 2001, 198(1/2/3) : 7-19.

[14] REN K F, GREHAN G, GOUESBET G. Prediction of reverse radiation pressure by generalized Lorenz-mie theory [ J ]. Applied Optics, 1996, 35 ( 15 ) : 27022710.

[15] SASAKI K, KOSHIOKA M, MISAWA H, et al. Optical trapping of a metal-particle and a water droplet by a scanning laser-beam $[\mathrm{J}]$. Applied Physics Letters, 1992, 60 (7) : 807-809.

[16] GRIFFITHS D J. Introduction to electrodynamics [M]. Englewood Cliffs, USA: Prentice-Hall, 1981.

[17] 曹志良, 梁言生, 严绍辉, 等. 不同偏振态光镊三维光 阱刚度的比较研究 $[\mathrm{J}]$. 光子学报, 2019, 48 (7): 0726002 .

CAO Zhiliang, LIANG Yansheng, YAN Shaohua, et al. Comparison investigation of the three-dimensional stiffness of optical tweezers with different polarization fields $[\mathrm{J}]$. Acta Photonica Sinica, 2019, 48 (7) : 0726002. (in Chinese)

[18 ] TONG L M, MILJKOVIC V D, ALIGNMENT K M. Alignment, rotation, and spinning of single plasmonic nanoparticles and nanowires using polarization dependent optical forces $[\mathrm{J}]$. Nano Letters, 2010, 10(1) : 268-273.

[19] RODRIGUEZ-OTAZO M, AUGIER-CALDERIN A, GALAUP J P, et al. High rotation speed of single molecular microcrystals in an optical trap with elliptically polarized light $[\mathrm{J}]$. Applied Optics, 2009, 48(14) : 2720-2730.

[20] SIMPSON S H, HANNA S. Optical trapping of spheroidal particles in gaussian beams $[\mathrm{J}]$. Journal of the Optical Society of America A: Optics, Image Science, and Vision, 2007, 24(2) : 430-443.

[21 ] BLIOKH K Y, RODRIGUEZ-FORTUNO F J, NORI F, et al. Spin-orbit interactions of light $[\mathrm{J}]$. Nature Photonics, 2015, 9(12): 796-808.

[22 ] PARKIN S, KNONER G, NIEMINEN T A, et al. Measurement of the total optical angular momentum transfer in optical tweezers [J]. Optics Express, 2006, 14(15) : 69636970.

[23 ] ORON R, BLIT S, DAVIDSON N, et al. The formation of laser beams with pure azimuthal or radial polarization [J]. Applied Physics Letters, 2000, 77 ( 21 ) : 33223324.

[24] CURTIS J E, GRIER D G. Structure of optical vortices [J]. Physical Review Letters, 2003, 90(13) : 133901.

[25] SIVILOGLOU G A, CHRISTODOULIDES D N. Accelerating finite energy airy beams $[\mathrm{J}]$. Optics Letters, 2007, $32(8)$ : 979-981.

[26] MIN Changjun, SHEN Zhe, SHEN Junfeng, et al. Focused plasmonic trapping of metallic particles $[\mathrm{J}]$. Nature Communications, 2013, 4 : 2891.

[27] WANG Xianyou, ZHANG Yuquan, DAI Yanmeng, et al. Enhancing plasmonic trapping with a perfect radially polarized beam [J]. Photonics Research, 2018, 6(9): 847-852. 
[28] ZHANG Yuquan, DOU Xiujie, DAI Yanmeng, et al. Alloptical manipulation of micrometer-sized metallic particles [J]. Photonics Research, 2018, 6(2) : 66-71.

[29] PADGETT M, BOWMAN R. Tweezers with a twist $[\mathrm{J}]$. Nature Photonics, 2011, 5(6) : 343-348.

[30 ] SIMPSON N B, DHOLAKIA K, ALLEN L, et al. Mechanical equivalence of spin and orbital angular momentum of light: an optical spanner $[\mathrm{J}]$. Optics Letters, $1997,22(1)$ : 52-54.

[31] ZHENG Zhu, ZHANG Baifu, CHEN Hao, et al. Optical trapping with focused airy beams $[\mathrm{J}]$. Applied Optics, 2011, 50(1) : 43-49.

[32 ] BAUMGARTL J, MAZILU M, DHOLAKIA K. Optically mediated particle clearing using airy wavepackets $[\mathrm{J}]$. Nature Photonics, 2008, 2(11) : 675-678.

[33 ] LI Manman, YAN Shaohui, YAO Baoli, et al. Optically induced rotation of rayleigh particles by vortex beams with different states of polarization $[\mathrm{J}]$. Physics Letters A, 2016, 380(1/2) : 311-315.

[34] LI Manman, YAN Shaohui, YAO Baoli, et al. Spinning and orbiting motion of particles in vortex beams with circular or radial polarizations $[\mathrm{J}]$. Optics Express, 2016, 24 (18) : 20604-20612.

[35 ] MAN Zhongsheng, DU Luping, ZHANG Yuquan, et al. Focal and optical trapping behaviors of radially polarized vortex beam with broken axial symmetry $[\mathrm{J}]$. AIP Advances, 2017, 7(6) : 4984813.

[36 ] BHEBHE N, WILLIAMS P A C, ROSALES-GUZMAN C, et al. A vector holographic optical trap $[\mathrm{J}]$. Scientific Reports, 2018, 8: 17387.

[37] GECEVICIUS M, DREVINSKAS R, BERESNA M, et al. Single beam optical vortex tweezers with tunable orbital angular momentum [J]. Applied Physics Letters, 2014, 104(23) : 231110.

[38 ] FALLMAN E, AXNER O. Design for fully steerable dualtrap optical tweezers $[\mathrm{J}]$. Applied Optics, 1997, 36 (10) : 2107-2113.

[39] MIO C, GONG T, TERRAY A, et al. Design of a scanning laser optical trap for multiparticle manipulation $[\mathrm{J}]$. Review of Scientific Instruments, 2000, 71 (5) : 21962200.

[40] CHU S, HOLLBERG L, BJORKHOLM J E, et al. 3dimensional viscous confinement and cooling of atoms by resonance radiation pressure $[\mathrm{J}]$. Physical Review Letters, $1985,55(1): 48-51$.

[41] CURTIS J E, KOSS B A, GRIER D G. Dynamic holographic optical tweezers $[\mathrm{J}]$. Optics Communications, $2002,207(1 / 2 / 3 / 4 / 5 / 6)$ : 169-175.
[42 ] SHAW L A, SPADACCINI C M, HOPKINS J B. Scanning holographic optical tweezers $[\mathrm{J}]$. Optics Letters, 2017, $42(15)$ : 2862-2865.

[43] KIRKHAM G R, BRITCHFORD E, UPTON T, et al. Precision assembly of complex cellular microenvironments using holographic optical tweezers $[\mathrm{J}]$. Scientific Reports, 2015, 5 : 8577.

[44] YU Xianghua, LI Runze, YAN Shaowei, et al. Experimental demonstration of $3 \mathrm{D}$ accelerating beam arrays $[\mathrm{J}]$. Applied Optics, 2016, 55(11) : 3090-3095.

[45] GARCES-CHAVEZ V, DHOLAKIA K, SPALDING G C. Extended-area optically induced organization of microparticies on a surface $[\mathrm{J}]$. Applied Physics Letters, 2005, 86 (3) : 031106.

[46] GU Min, HAUMONTE J B, MICHEAU Y, et al. Laser trapping and manipulation under focused evanescent wave illumination $[\mathrm{J}]$. Applied Physics Letters, 2004, 84 (21) : 4236-4238.

[47] GU Min, KE Puchun. Depolarization of evanescent waves scattered by laser-trapped gold particles: effect of particle size [J]. Journal of Applied Physics, 2000, 88 (9) : 5415-5420.

[48] KURIAKOSE S, GAN Xiasong, CHON J W M, et al. Optical lifting force under focused evanescent wave illumination: a ray optics model $[\mathrm{J}]$. Journal of Applied Physics, 2005, 97(8) : 083103

[49] KAWATA S, SUGIURA T. Movement of micrometer-sized particles in the evanescent field of a laser-beam $[\mathrm{J}]$. Optics Letters, 1992, 17(11) : 772-774.

[50 ] NG L N, ZERVAS M N, WILKINSON J S, et al. Manipulation of colloidal gold nanoparticles in the evanescent field of a channel waveguide $[\mathrm{J}]$. Applied Physics Letters, 2000, 76(15) : 1993-1995.

[51] RIGHINI M, ZELENINA A S, GIRARD C, et al. Parallel and selective trapping in a patterned plasmonic landscape [J]. Nature Physics, 2007, 3(7) : 477-480.

[52] ZHANG Weihua, HUANG Lina, SANTSCHI C, et al. Trapping and sensing $10 \mathrm{~nm}$ metal nanoparticles using plasmonic dipole antennas $[\mathrm{J}]$. Nano Letters, 2010, 10 (3) : 1006-1011.

[53 ] LIN Y C, LEE P T. Efficient optical trapping and detection of nanoparticle via plasmonic bowtie notch $[\mathrm{J}]$. IEEE Photonics Journal, 2019, 11(2) : 4800610.

[54] SHOJI T, SAITOH J, KITAMURA N, et al. Permanent fixing or reversible trapping and release of DNA micropatterns on a gold nanostructure using continuous-wave or femtosecond-pulsed near-infrared laser light $[\mathrm{J}]$. Journal of the American Chemical Society, 2013, 135 ( 17 ): 
6643-6648.

[55] WANG Xianyou, DAI Yanmeng, ZHANG Yuquan, et al. Plasmonic manipulation of targeted metallic particles by polarization-sensitive metalens [ J ]. ACS Photonics, $2018,5(7)$ : 2945-2950.

[56] MAN Zhongsheng, ZHANG Shuoshuo, BAI Zhidong, et al. All-optical and dynamic manipulation of surface plasmon polaritons by tailoring the polarization state of incident light $[\mathrm{J}]$. Laser Physics Letters, 2019, 16(2) : 026001.

[57] ZHANG Yuquan, SHI Wei, SHEN Zhe, et al. A plasmonic spanner for metal particle manipulation $[\mathrm{J}]$. Scientific Reports, 2015, 5: 15446.

[58] ZHANG Yuquan, WANG Jian, SHEN Junfeng, et al. Plasmonic hybridization induced trapping and manipulation of a single $\mathrm{Au}$ nanowire on a metallic surface $[\mathrm{J}]$. Nano Letters, 2014, 14(11) : 6430-6436.

[59] LI Zhipeng, ZHANG Shunping, TONG Lianming, et al. Ultrasensitive size-selection of plasmonic nanoparticles by fano interference optical force $[\mathrm{J}]$. ACS Nano, 2014, 8 (1) : 701-708

[60 ] PAIVA J S, JORGE P S, ROSA C C, et al. Optical fiber tips for biological applications: from light confinement, biosensing to bioparticles manipulation $[\mathrm{J}]$. Biochimica et Biophysica Acta-General Subjects, 2018, 1862 ( 5 ) : 1209-1246.

[61] RIBEIRO R R, SOPPERA O, OLIVA A G, et al. New trends on optical fiber tweezers $[\mathrm{J}]$. Journal of Lightwave Technology, 2015, 33(16) : 3394-3405.

[62] RICKELT L F, OTTOSEN L D M, KUHL M. Etching of multimode optical glass fibers: a new method for shaping the measuring tip and immobilization of indicator dyes in recessed fiber-optic microprobes $[\mathrm{J}]$. Sensors and Actuators B: Chemical, 2015, 211 : 462-468.

[63 ] VAIANO P, CAROTENUTO B, PISCO M, et al. Lab on fiber technology for biological sensing applications $[\mathrm{J}]$. Laser \& Photonics Reviews, 2016, 10(6) : 922-961.

[64] ZHU Wei, SHI Tielin, TANG Zirong, et al. Dynamic selective etching: a facile route to parabolic optical fiber nano-probe $[\mathrm{J}]$. Optics Express, 2013, 21 (6) : 69196927.

[65] XIN Hongbao, LI Baojun. Fiber-based optical trapping and manipulation $[\mathrm{J}]$. Frontiers of Optoelectronics, 2019, 12(1): 97-110.

[66] YUAN Libo, LIU Zhihai, YANG Jun, et al. Twin-core fiber optical tweezers $[\mathrm{J}]$. Optics Express, 2008, 16 (7) : 4559-4566.

[67] LI Yuchao, XIN Hongbao, LIU Xiaoshuai, et al. Trapping and detection of nanoparticles and cells using a parallel photonic nanojet array $[\mathrm{J}]$. ACS Nano, 2016, 10(6) : 5800-5808.

[68 ] LU Jinsheng, YANG Hangbo, ZHOU Lina, et al. Lightinduced pulling and pushing by the synergic effect of optical force and photophoretic force $[\mathrm{J}]$. Physical Review Letters, 2017, 118(4) : 043601.

[69] CHEN Jiajie, CONG Hengi, LOO F C, et al. Thermal gradient induced tweezers for the manipulation of particles and cells $[J]$. Scientific Reports, 2016, 6: 35814.

[70 ] LIN Linhan, PENG Xiaolei, ZHENG Yuebing. Reconfigurable opto-thermoelectric printing of colloidal particles [J]. Chemical Communications, 2017, 53 (53) : 73577360.

[71 ] LIU Yaoran, LIN Linhan, RAJEEVA B B, et al. Nanoradiator-mediated deterministic opto-thermoelectric manipulation [J]. ACS Nano, 2018, 12(10): 10383-10392.

[72] KOTNALA A, ZHENG Yuebing. Opto-thermophoretic fiber tweezers [J]. Nanophotonics, 2019, 8(3): 475-485.

[73 ] KOLLIPARA P S, LIN Linhan, ZHENG Yuebing. Thermoelectro-mechanics at individual particles in complex colloidal systems $[\mathrm{J}]$. Journal of Physical Chemistry C, 2019, $123(35)$ : 21639-21644.

[74] LIN Linhan, WANG Mingsong, PENG Xiaolei, et al. Opto-thermoelectric nanotweezers $[\mathrm{J}]$. Nature Photonics, 2018, 12(4) : 195-201.

[75] KOTSIFAKI D G, KANDYLA M, LAGOUDAKIS P G. Plasmon enhanced optical tweezers with gold-coated black silicon [J]. Scientific Reports, 2016, 6: 26275.

[76] ROXWORTHY B J, TOUSSAINT K C. Femtosecondpulsed plasmonic nanotweezers $[\mathrm{J}]$. Scientific Reports, 2012, 2: 660.

[77] SHAKHOV A M, ASTAFIEV A A, PLUTENKO D O, et al. Femtosecond optical trap-assisted nanopatterning through microspheres by a single Ti: Sapphire oscillator [J]. Journal of Physical Chemistry C, 2015, 119(22): 12562-12571.

[78 ] GU Bin, CAO Xi, RUI Guanghao, et al. Vector beams excited nonlinear optical effects $[\mathrm{J}]$. Journal of Nonlinear Optical Physics \& Materials, 2018, 27(4) : 1850045.

[79] GONG Liping, ZHANG Xiaohe, GU Bing, et al. Optical pulling forces on rayleigh particles using ambient optical nonlinearity $[\mathrm{J}]$. Nanophotonics, 2019, 8 (6) : 11171124.

[80] JIANG Yuqiang, NARUSHIMA T, OKAMOTO H. Nonlinear optical effects in trapping nanoparticles with femtosecond pulses [J]. Nature Physics, 2010, 6(12) : 10051009.

[81] ZHANG Yuquan, SHEN Junfeng, MIN Changjun, et al. 
Nonlinearity-induced multiplexed optical trapping and manipulation with femtosecond vector beams $[\mathrm{J}]$. Nano Letters, 2018, 18(9) : 5538-5543.

[82 ] CHEN Jun, NG J, LIN Zhifang, et al. Optical pulling force $[\mathrm{J}]$. Nature Photonics, 2011, 5(9) : 531-534.

[83] SAENZ J J. Optical forces laser tractor beams $[\mathrm{J}]$. Nature Photonics, 2011, 5(9) : 514-515.

[84 ] CUCHE A, STEIN B, CANAGUIER D A, et al. Brownian motion in a designer force field: dynamical effects of negative refraction on nanoparticles $[\mathrm{J}]$. Nano Letters, 2012 , $12(8)$ : 4329-4332.

[85] RODRIGUEZ-FORTUNO F J, ENGHETA N, MARTINEZ A, et al. Lateral forces on circularly polarizable particles near a surface $[\mathrm{J}]$. Nature Communications, 2015, 6: 10263.

[86] SUKHOV S, KAJORNDEJNUKUL V, NARAGHI R R, et al. Dynamic consequences of optical spin-orbit interaction [J]. Nature Photonics, 2015 , 9(12) : 809-812.

[87] WANG S B, CHAN C T. Lateral optical force on chiral particles near a surface $[\mathrm{J}]$. Nature Communications, 2014, $5: 3307$.

[88 ] ZHONG Mincheng, WEI Xunbin, ZHOU Jinhua, et al. Trapping red blood cells in living animals using optical tweezers $[\mathrm{J}]$. Nature Communications, 2013, 4: 1768.

[89] ZHANG Yuquan, WU Xiaojing, MIN Changjun, et al. Engineered tumor cell apoptosis monitoring method based on dynamic laser tweezers $[\mathrm{J}]$. Biomed Research International, 2014, 2014: 279408

[90] WANG M D, YIN H, LANDICK R, et al. Stretching DNA with optical tweezers $[\mathrm{J}]$. Biophysical Journal, 1997, 72(3) : 1335-1346.

[91] PANG Yuanjie, GORDON R. Optical trapping of a single protein [J]. Nano Letters, 2012, 12(1) : 402-406.

[92 ] SVEDBERG F, LI Zhipeng, XU Hongxing, et al. Creating hot nanoparticle pairs for surface-enhanced Raman spectroscopy through optical manipulation $[\mathrm{J}]$. Nano Letters, 2006, 6(12) : 2639-2641.

[93] SHEN Junfeng, WANG Jian, ZHANG Cuijiao, et al. Dynamic plasmonic tweezers enabled single-particle-filmsystem gap-mode surface-enhanced Raman scattering [J]. Applied Physics Letters, 2013, 103(19) : 191119.
[94] SUN Qiuyue, ZHANG Yuquan, SUN Lixun, et al. Microscopic surface plasmon enhanced Raman spectral imaging [J]. Optics Communications, 2017, 392: 64-67.

[95 ] YANG Aiping, DU Luping, DOU Xiujie, et al. Sensitive gap-enhanced Raman spectroscopy with a perfect radially polarized beam [J]. Plasmonics, 2018, 13 (3) : 991996.

[96] ZHANG Yuquan, SHEN Junfeng, XIE Zhenwei, et al. Dynamic plasmonic nano-traps for single molecule surfaceenhanced Raman scattering [ J ]. Nanoscale, 2017, 9 (30) : 10694-10700.

[97 ] BRADAC C. Nanoscale optical trapping: a review [ J ]. Advanced Optical Materials, 2018, 6(12) : 1800005.

[98 ] RAJEEVA B B, LIN Linhan, PERILLO E P, et al. Highresolution bubble printing of quantum dots $[\mathrm{J}]$. ACS Applied Materials \& Interfaces, 2017, 9 (19) : 16725 16733.

[99] MONTELONGO Y, YETISEN A K, BUTT $\mathrm{H}$, et al. Reconfigurable optical assembly of nanostructures $[\mathrm{J}]$. Nature Communications, 2016, 7: 12002.

[100] DO J, FEDORUK M, JACKEL F, et al. Two-color laser printing of individual gold nanorods $[\mathrm{J}]$. Nano Letters, $2013,13(9)$ : 4164-4168.

[101] LIN Linhan, PENG Xiaolei, MAO Zhangming, et al. Bubble-pen lithography $[\mathrm{J}]$. Nano Letters, 2016, 16 (1) : 701-708.

[102] SMALLEY D E, NYGAARD E, SQUIRE K, et al. A photophoretic-trap volumetric display $[\mathrm{J}]$. Nature, 2018, 553(7689) : 486-490.

[103] JURADO-SANCHEZ B, ESCARPA A. Milli, micro and nanomotors: novel analytical tools for real-world applications $[\mathrm{J}]$. Trac-Trends in Analytical Chemistry, 2016, 84 : 48-59.

[104] GALAJDA P, ORMOS P. Complex micromachines produced and driven by light $[\mathrm{J}]$. Applied Physics Letters, 2001, 78(2) : 249-251.

[105] QUINTO-SU P A. A microscopic steam engine implemented in an optical tweezer $[\mathrm{J}]$. Nature Communications, 2014,5 : 5889

【中文责编：方 圆; 英文责编：溯 心】 